\title{
Pontryagin's Minimum Principle based Model Predictive Control of Energy Management for a Plug-In Hybrid Electric Bus
}

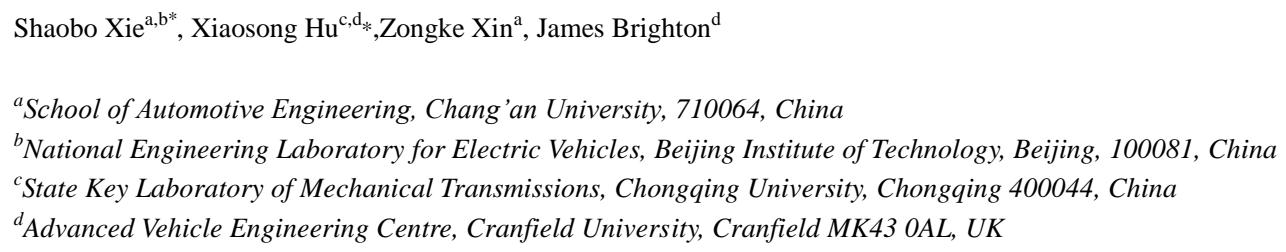

*X. Hu and S. Xie equally contributed to this research work. Corresponding author: Email: xiaosonghu@ieee.org and xieshaobo@ chd.edu.cn

\begin{abstract}
A B S T RA C T:
To improve computational efficiency of energy management strategies for plug-in hybrid electric vehicles (PHEVs), this paper proposes a stochastic model predictive controller (MPC) based on Pontryagin's Minimum Principle (PMP), which differs from widely used dynamic programming (DP)-based predictive methods. First, short-time speed forecasting is achieved using a Markov chain model, based on real-world driving cycles. The PMP- and DP-based MPCs are compared under four preview horizons $(5 \mathrm{~s}, 10 \mathrm{~s}, 15 \mathrm{~s}$ and $20 \mathrm{~s})$, and the results show that the computational time of the DP-MPC is almost four times of that in the PMP-MPC. Moreover, the influence of predication horizon length on computational time and energy consumption is examined. Given a preview horizon of 5s, the PMP-MPC holds a total energy consumption cost of 7.80 USD and computational time per second of $0.0130 \mathrm{~s}$. When the preview horizon increases to $20 \mathrm{~s}$, the total cost is 7.77 USD with the computational time per second increasing to $0.0502 \mathrm{~s}$. Finally, DP, PMP, and rule-based strategies are contrasted to the PMP-MPC method, further demonstrating the promising performance and computational efficiency of the proposed methodology.
\end{abstract}

\section{Keywords:}

Plug-in hybrid electric bus;

Stochastic model predictive control;

Pontryagin's Minimum Principle;

Dynamic programming;

Algorithmic efficiency 


\section{Introduction}

\subsection{Literature review}

The past few decades have witnessed a surging trend towards electrified vehicles worldwide, with the aim of reducing carbon footprints, saving energy, and from a technology perspective, upgrading the automotive sector [1-2]. In particular, plug-in hybrid electric vehicles, which are capable of absorbing electricity from the grid, can enhance fuel economy, reduce exhaust emissions, and overcome range anxiety associated with long-distance travels [3].

In general, developing power management strategies for effectively coordinating multiple energy sources of hybrid powertrains, such as the engine and energy storage system (electrochemical battery and/or ultra-capacitor), will be indispensable in achieving improved fuel economy [4]. Furthermore, to meet the requirement of real-time applications, proposed strategies must also be time-efficient. Thus far, a number of power management strategies have been suggested including rule-based and fuzzy logic-based strategies [5-6], optimal control theory methods, such as global optimization [7-8] and instantaneous optimization [9], as well as a variety of evolutionary algorithms, including particle swarm optimization [10], artificial neural network [11], simulated annealing [12], and reinforcement learning [13].

Dynamic programming has been extensively utilized in the development of energy management strategies (EMSs), ensuring a global optimality of both hybrid electric vehicles (HEVs) [14-15] and plug-in hybrid electric vehicles [2, 16]. Dynamic programming has become the benchmark for evaluating all other strategies. However, the method itself is computationally intensive, due to the massive number of interpolations required to estimate the cost-to-go values of discrete points at each state and for each input variable. To achieve global optimization, stochastic DP has been proposed [17-18]. Furthermore, Pontryagin's Minimum Principle is another popular global optimization algorithm [19-20]. With PMP, the Hamiltonian function is instantaneously minimized while satisfying the state of charge (SOC) boundary constraints and other physical constraints [21-22]. However, both the DP and PMP algorithms can only be carried out if the complete information of a running cycle is known. Moreover, online applications cannot be realized.

Besides PMP, the Equivalent Consumption Minimum Strategy (ECMS) has also been introduced to develop an online EMS [23]. Novel application-oriented ECMSs, such as adaptive [24] and map-based ECMS [2, 25], or artificial neural network-enhanced EMCS were further proposed [11], in which the equivalent factor is dynamically tuned based on environmental information.

Although DP and PMP methods cannot be implemented in EMSs without prior knowledge of the driving cycle, this can be overcome by taking advantage of speed forecasting [26-27] or driving pattern recognition [28-29]. Model predictive control is then achieved by embedding DP or PMP method into a moving horizon. An exponential receding model was previously proposed to realize predictive control, where the required torque sequence was considered to decline exponentially [30]. In addition, the use of stochastic MPC in HEVs and PHEVs has recently attracted attention [31-33], and future speed or power requirement sequences were predicted using the Markov chain [34-35]. At the same time, MPC strategies are being developed using artificial neural networks to conduct speed forecasting [26, 36-37]. Moreover, a cascade control concept within the MPC strategy was reported for a parallel HEV. Using this strategy, the discharge path can be optimized by the master controller while the salve controller applies power from the master controller to the powertrain [38]. Similarly, two-level model predictive control was suggested for minimizing the lap time of hybrid race cars [39]. Moreover, MPC has been demonstrated for network-connected environments, such as a vehicle-to-vehicle (V2V) and vehicle-to-infrastructure (V2I) communications or intelligent transport systems (ITS) [40].

To improve real-time implementability of EMSs, explicit model predictive control (MPC) was introduced by simplifying a control model with piecewise-affine functions so as to decrease computational burden [41-42]. In addition, a generalized minimum residual was proposed to solve issues associated with online iterative optimal algorithms, thereby achieving efficient control [43].

Despite the availability of various predictive power management methods, DP is still widely chosen over receding horizon functions for solving optimization problems for both HEVs and PHEVs [26, 30-31, 35-37, 44-47]. For example, DP-based MPC (DP-MPC) was applied to a co-axis serial-parallel PHEV, with the consideration of three constrained methods to restrain the SOC boundary value [35]. Moreover, better speed prediction accuracy was achieved by incorporating the Monte Carlo Markov chain into MPC [44]. Meanwhile, a modified DP-MPC was suggested by 
classifying driving behaviors with $K$-means [45]. A novel scholastic MPC combining online learning with the Markov chain was introduced to account for variations in driver behavior [47]. However, the PMP and ECMS methods embedded in MPC, were mostly applied to power management of HEVs [37, 48]. It is worth noting that MPC schemes for HEVs and PHEVs are highly different. The SOC trace of an HEV always remains stable, so that the reference SOC can be set as a constant level, making it easier to solve the optimization problem. Instead, the SOC of PHEVs varies with environmental conditions such as driving distance. Therefore, MPC of PHEVs requires a dynamic tuning of the equivalent factor for ECMS and co-state for PMP in a rolling horizon. Thus, predictive EMSs implemented in HEVs, in particular, for PMP-based methods, are not trivially transferrable to PHEVs.

In addition to improving fuel economy, another objective of power management is to extend the lifespan and improve the charge capacity of batteries. As for multi-objective optimization problems, approaches like convex optimization were often used [49], as well as algorithms that can cope with conflicting factors, such as the non-dominated Sorting Genetic Algorithm-II (NSGA-II) [50].

\subsection{Motivation for this work}

Despite clear advantages of predictive strategies [31-33, 35-37, 46-48], a number of issues merit further exploration.

First, when using common optimization algorithms, such as DP and PMP, to develop predictive EMSs, existing studies mainly focused on minimizing total energy consumption or cost, with a limited emphasis on computational efficiency. The efficiency of optimization algorithms operating over consecutive prediction horizons should be considered in priority, particularly when designing online EMSs, in order to lower controller hardware requirements and reduce production costs. The PMP method has been combined with receding horizon control to generate a MPC strategy for HEVs [48]. To the best of the authors' knowledge, a PMP-based MPC (PMP-MPC) with a detailed numerical solution for PHEVs and a comparison of DP-MPC and PMP-MPC, in terms of computational efficiency, are still lacked in the literature.

Second, although ECMS is considered as an effective approach for implementing MPC, this method is derived from PMP by assuming that the derivative of the co-state variable is zero, thus neglecting battery dynamics [48]. To some extent, its solution precision is lowered. Furthermore, in the ECMS-based MPC, a dynamic modification of the equivalent factor over each horizon to achieve near-optimal fuel consumption is challenging. This will most likely induce complicated issues, versus PMP-based methods, particularly for PHEVs.

\subsection{Contributions of this work}

The main contributions of this work are highlighted as follows. (1) A general framework of PMP-MPC is proposed for PHEVs. The PMP-MPC method differs from other widely used ECMS-based predictive energy management strategies that require tuning of the equivalent factor using a variety of complicated approaches [23]. (2) The computational efficiency of PMP-MPC is significantly better than that of DP-MPC, suggesting that it may be a favorable algorithm for developing time efficiency-conscious predictive energy management strategies. (3) The Markov chain-based speed predicator is incorporated into the predictive method to yield an online stochastic MPC strategy, resulting in improved fuel economy, in contrast to rule-based control strategies.

\subsection{Organization of this paper}

The remainder of this paper is organized as follows. Section 2 presents the architecture of PMP-MPC and Section 3 describes the modeling of the powertrain. In Section 4, speed forecasting is implemented based on the Markov chain model, and in Section 5, a reference SOC planning is given. Then, PMP-MPC is formulated in Section 6, and the shooting method, to realize a numerical solution of the model, is also discussed. Section 7 introduces the DP-MPC for a comparison with PMP-MPC, and results of different methods are discussed and analyzed. Finally, conclusions are summarized in Section 8.

\section{Architecture of PMP-MPC}

A diagram outlining the proposed PMP-MPC architecture is presented in Fig. 1, including the research process and techniques used for solving each step in the process. 


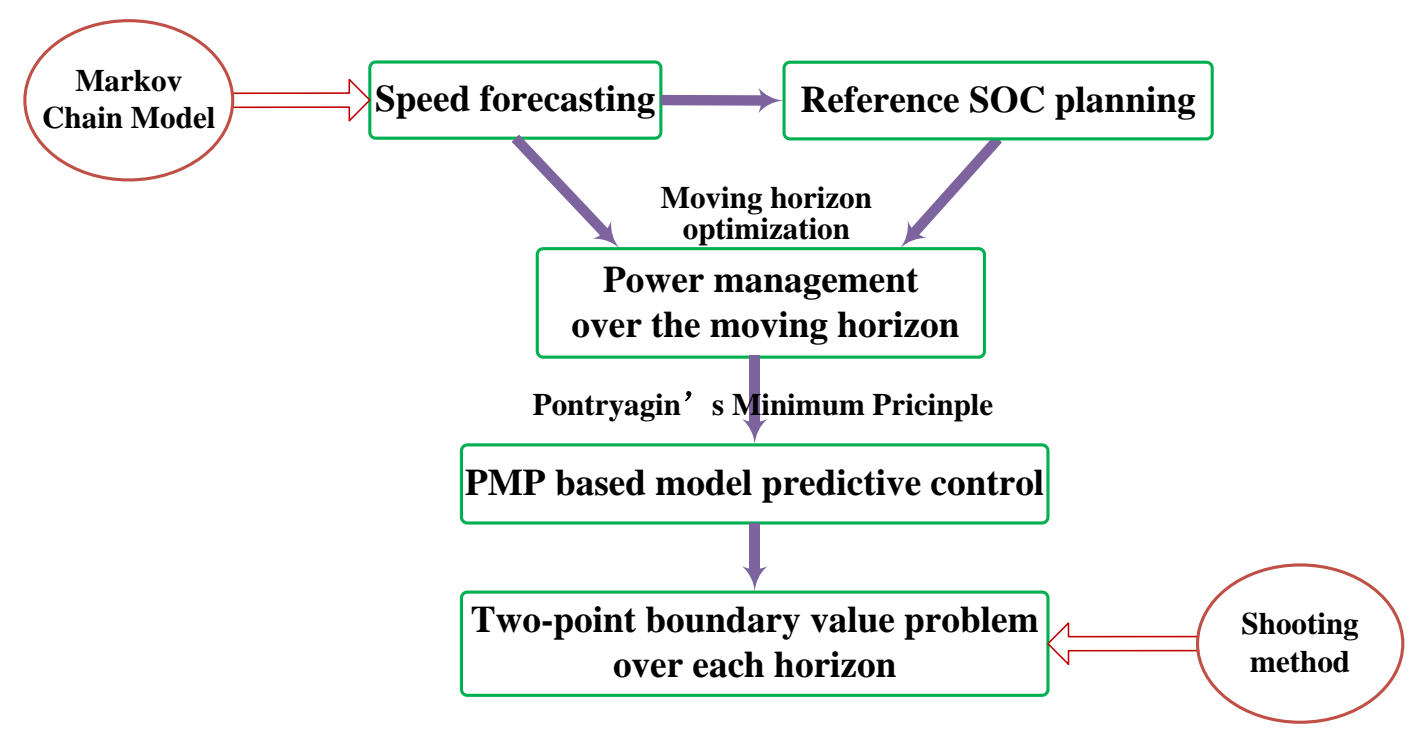

Fig. 1. Architecture of the proposed PMP-MPC.

The aim of MPC is to optimize power management over a moving horizon. Therefore, speed forecasting using Markov chain model can be leveraged to supply the preview power requirements. Reference SOC planning defines the upper and lower boundaries of the SOC values in each horizon for which the optimization algorithm, either DP or PMP, must be implemented, thus guiding when the battery discharges to achieve better fuel economy. The PMP method was chosen, as it can be used to obtain the local solution of each horizon, yielding PMP-MPC, and can improve computational efficiency of MPC. Numerically, this type of PMP-MPC results in a two-point boundary value problem that can be solved using the shooting method.

\section{Powertrain modeling}

\subsection{Powertrain description}

Fig. 2 illustrates the powertrain of the plug-in hybrid electric bus (PHEB) used in this study, which features a typical serial structure. A natural gas engine is mechanically connected to an integrated starter and generator (ISG) to form an engine-generator-unit (EGU). A special two-independent-motor driving system compresses the driveline, as shown in Fig. 3. Figure 4 depicts the fuel consumption rate of the engine and its brake specific fuel consumption (BSFC). Based on the experimentally verified data including the rotational speed, torque, and electrical power, the three-dimensional and planar efficiency maps of the generator and the motor are portrayed in Fig. 5 and Fig. 6, respectively. The main parameters are summarized in Table 1.

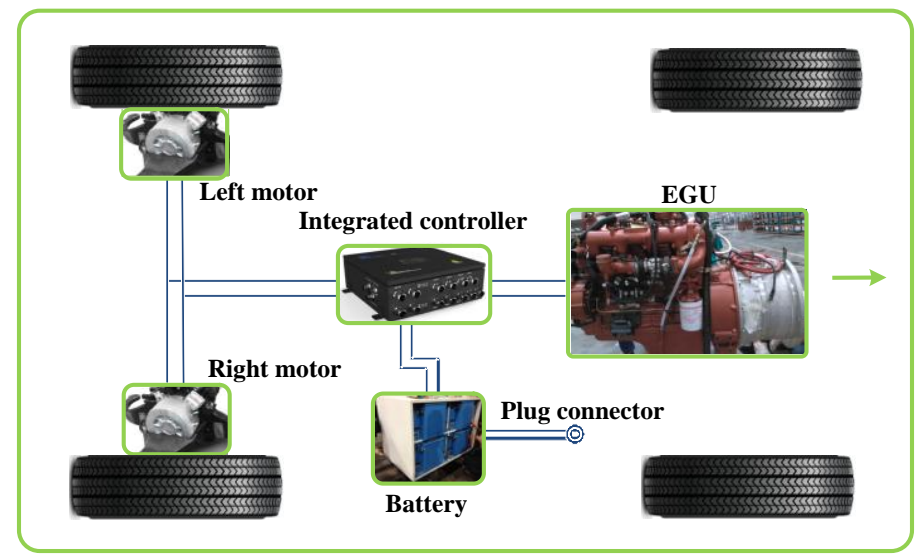

Fig. 2. Powertrain architecture of plug-in hybrid electric bus [51]. 


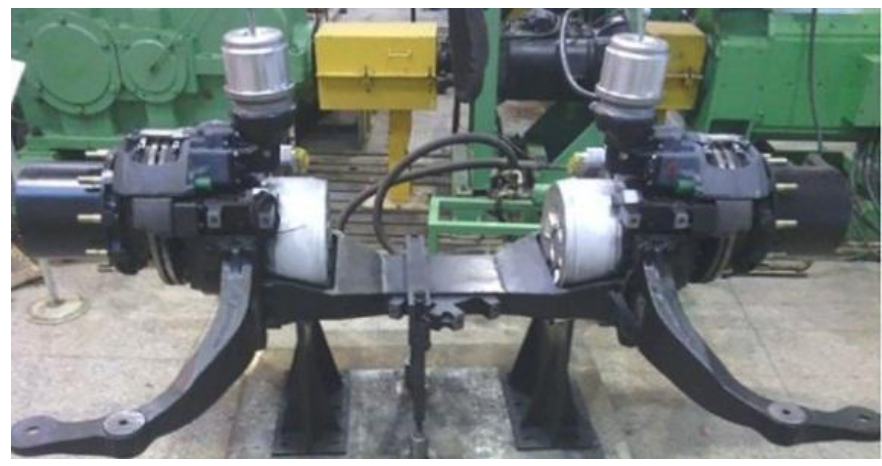

Fig. 3. Two-independent-motor driving system [51].

Table 1 Specification of PHEB [46]

\begin{tabular}{ll}
\hline Item & Description \\
\hline Vehicle & Curb weight: $13500 \mathrm{~kg}$; Two-level ratio: 13.9 \\
\hline \multirow{2}{*}{ Engine } & Natural gas engine; Displacement: $4.2 \mathrm{~L} ;$ \\
& Rated power: $88 \mathrm{~kW}$; Max rotational speed: $2800 \mathrm{rpm}$ \\
\hline \multirow{2}{*}{ ISG } & Permanent magnetic motor; Max power: $130 \mathrm{~kW} ;$ \\
& Max torque: $500 \mathrm{Nm}$; Max rotational Speed: $6000 \mathrm{rpm}$ \\
\hline \multirow{2}{*}{ Tractor motor } & $\begin{array}{l}\text { Permanent magnetic motor; Max power: } 150 \mathrm{~kW} ; \\
\\
\text { Max torque: } 650 \mathrm{Nm} \text {; Max rotational speed: } 6000 \mathrm{rpm}\end{array}$ \\
\hline \multirow{2}{*}{ Battery } & Lithium-iron phosphate battery; Nominal capacity: $120 \mathrm{Ah} ;$ \\
& Total voltage: $537.6 \mathrm{~V}$ \\
\hline
\end{tabular}

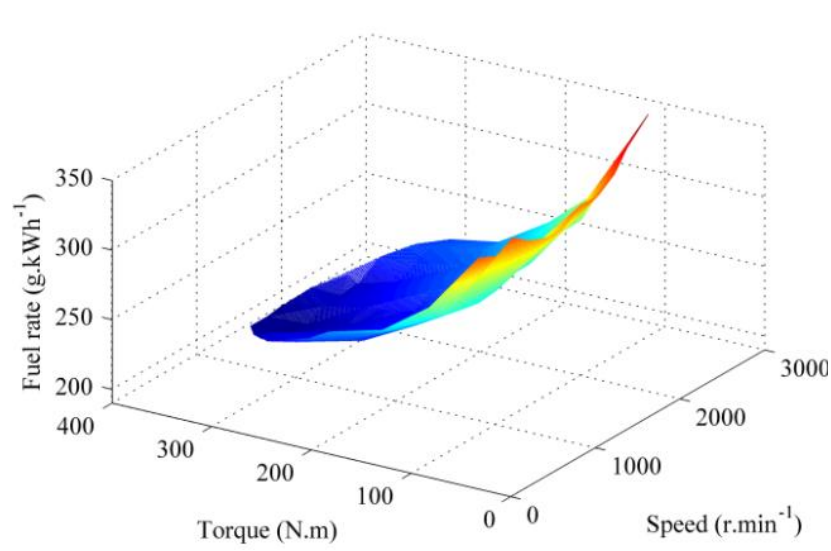

(a) Fuel rate of the natural gas engine

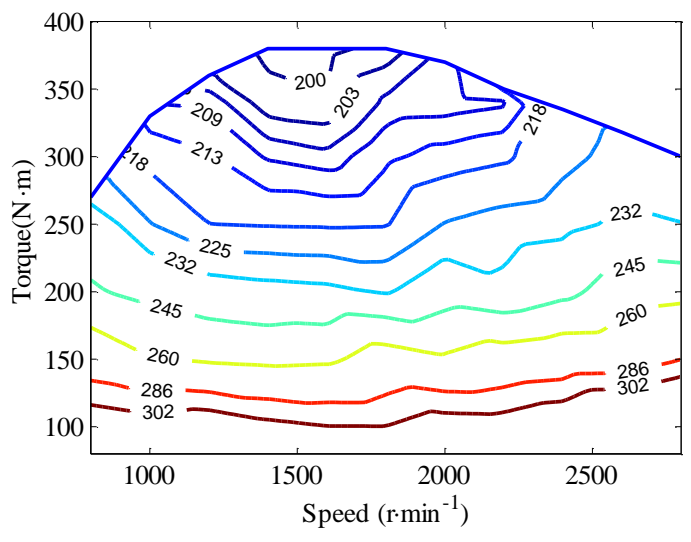

(b) BSFC of the natural gas engine

Fig. 4. Efficiency of the natural gas engine [46].

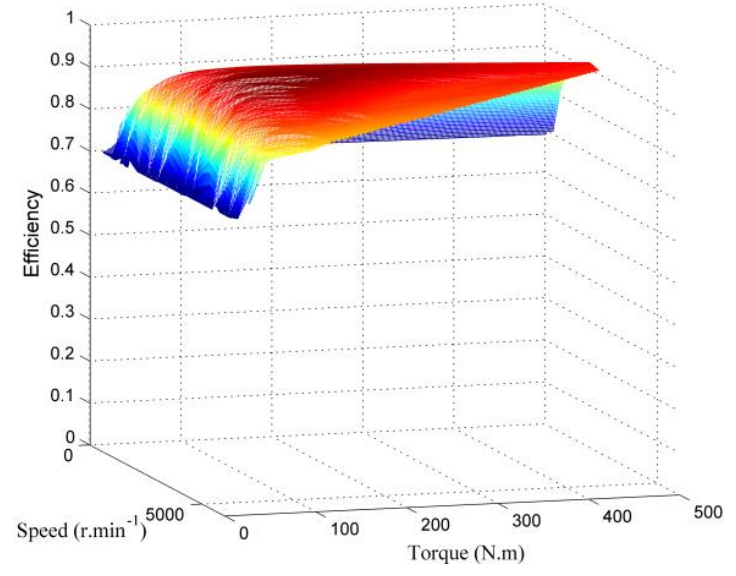

(a) Three-dimensional efficiency of the generator

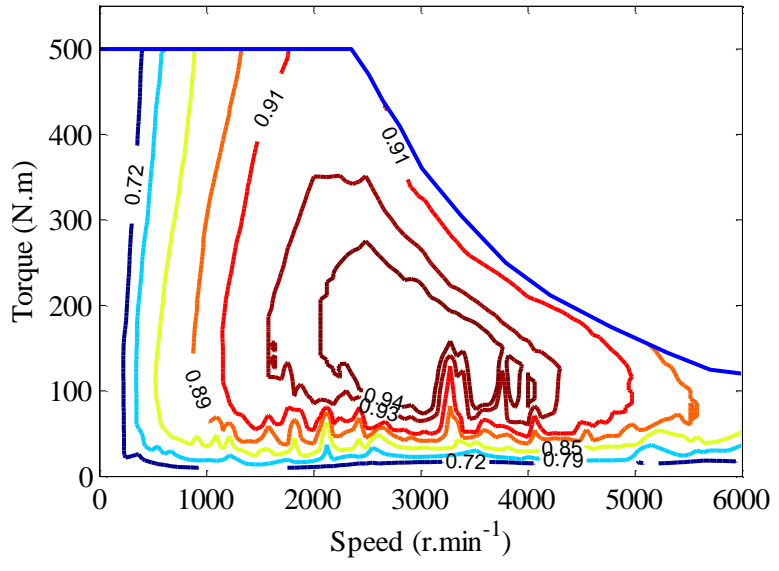

(b) Planar efficiency of the generator

Fig. 5. Efficiency of the generator [46]. 


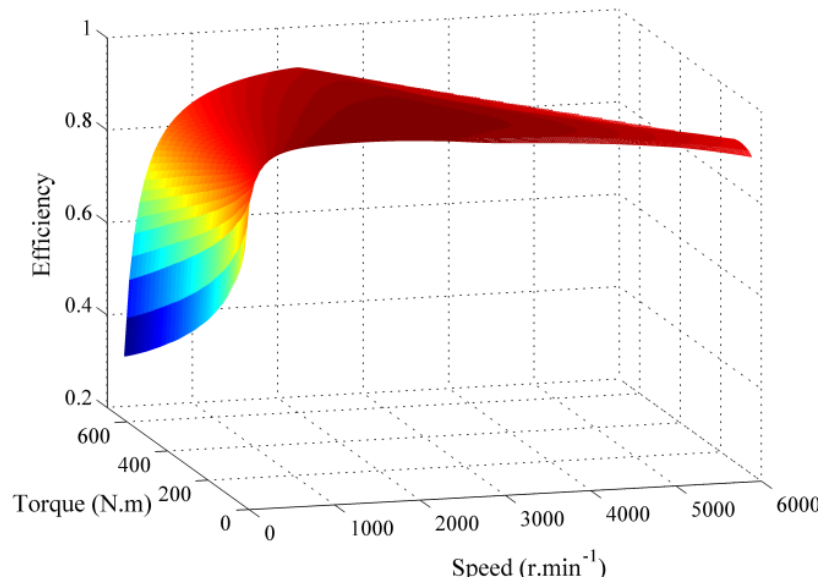

(a) Three-dimensional efficiency of the driving motor

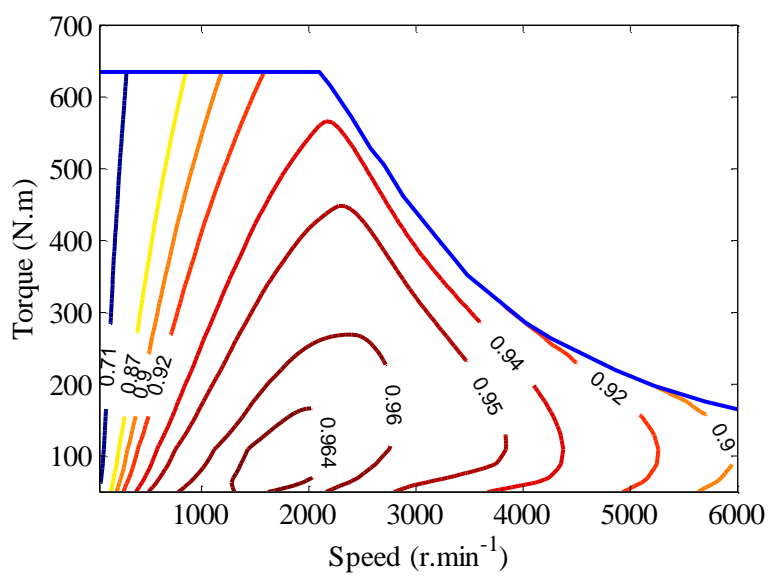

(b) Planar efficiency of the driving motor

Fig. 6. Efficiency of the driving motor [46].

\subsection{Battery model}

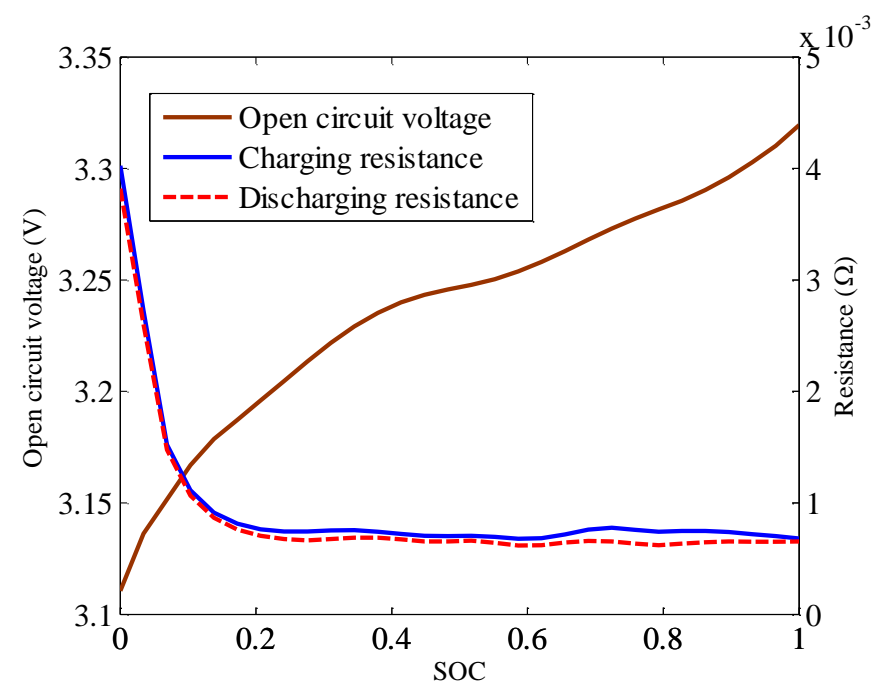

Fig. 7. Open circuit voltage and internal resistance with respect to SOC of a single battery cell [23].

The energy storage system is a lithium iron phosphate battery, and the battery pack contains 168 cells with a nominal capacity of $120 \mathrm{Ah}$ and total voltage of $537.6 \mathrm{~V}$. An equivalent internal resistance model is employed to simulate the battery circuit, in which the open circuit voltage and equivalent internal resistance are parametric functions of the battery SOC [52]. Also, it is assumed that the state of health, state of power and thermal effect are neglected for the battery system. Based on selected experimental data, the estimated open circuit voltage and internal resistance with respect to SOC for each cell are shown in Fig. 7.

Taking into account internal electrical loss, the power balance equation for the battery system is governed by

$$
P_{b a t}=P_{b}+P_{l}=P_{b}+I^{2} R_{b}
$$

where $P_{b a t}$ is the total power going into or out of the battery, $P_{b}$ is the power at the terminals, $P_{l}$ represents the internal loss of the battery, $I$ is the electrical current and $R_{b}$ is the equivalent internal resistance.

\subsection{Vehicle dynamics model}

For the PHEB with a two-independent-motor driving system, the force balance equation can be given as

$$
m g f+\frac{C_{d} A}{21.15} v^{2}+\delta m \frac{d v}{d t}=\frac{\left(T_{m 1} n_{m 1}+T_{m 2} n_{m 2}\right) \eta_{m}^{\operatorname{sgn}\left(T_{m 1}\right)} i_{0}}{r}
$$

where $m$ is the curb mass, $g$ is the gravitational acceleration, $f$ is the rolling resistance coefficient, $C_{d}$ is the air resistance coefficient, $A$ is the front area, $v$ is the vehicle speed, $\delta$ is the equivalent mass moment of inertia, $i_{0}$ is the final 
gear ratio, $r$ is the tire radius; $T_{m 1}, T_{m 2}$ and $n_{m 1}, n_{m 2}$ are the output motor torques and rotational speeds of the two sides, $m 1$ and $m 2$, respectively, and $\eta_{m}$ is the mechanical efficiency of the final drive. If $T_{m 1}$ is positive (in a propelling mode), the value of $\operatorname{sgn}$ equals 1 , or its value is -1 . Moreover, the power balance equation can be expressed as

$$
P_{b}+P_{e g u}=P_{m 1}+P_{m 2}+P_{a u x}
$$

where $P_{e g u}$ is the EGU output power, $P_{m 1}$ and $P_{m 2}$ are the motor output electric power of the two sides, and $P_{a u x}$ is the power consumption of auxiliary components, e.g., the steering system and braking system.

Moreover, since the main controller always delivers the same torque and power commands to the two driving motors, both motors are assumed to be in the same dynamic state, that is,

$$
\left\{\begin{array}{l}
T_{m 1}=T_{m 2} \\
P_{m 1}=P_{m 2}
\end{array}\right.
$$

\section{Speed forecasting}

\subsection{Markov chain forecaster}

In general, speed forecasting methods include data-driven methods (e.g., artificial neural network and Markov chain) and model-based methods (e.g., exponential receding model). The Markov chain model takes into account the randomness of speed variation and therefore provides superior forecasting results, particularly in terms of computational efficiency [46]. In this study, the speed forecaster is established using this method. To further improve the reliability of predictions, real-world driving data were sampled and used to calculate a probabilistic transition model rather than standard driving schedules such as the Chinese City Bus Driving Cycle (CCBDC) [35], Urban Dynamometer Driving Schedule (UDDS) [48].

The city bus route investigated in this study includes a round trip with a total distance of approximately $70 \mathrm{~km}$. Seven speeding profiles were sampled to implement the speed forecasting, as shown in Fig. 8 [46]. The first six were used to generate the probability transition matrix, and the remaining profile for verification.

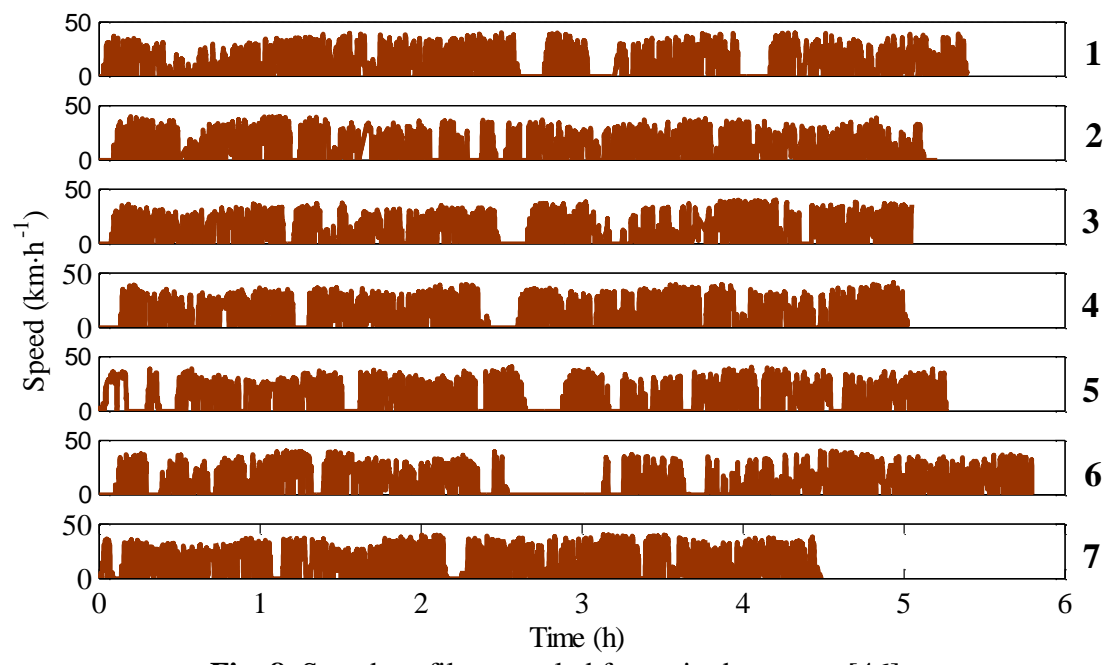

Fig. 8. Speed profiles sampled from city bus route [46].

\subsection{Forecasting results}




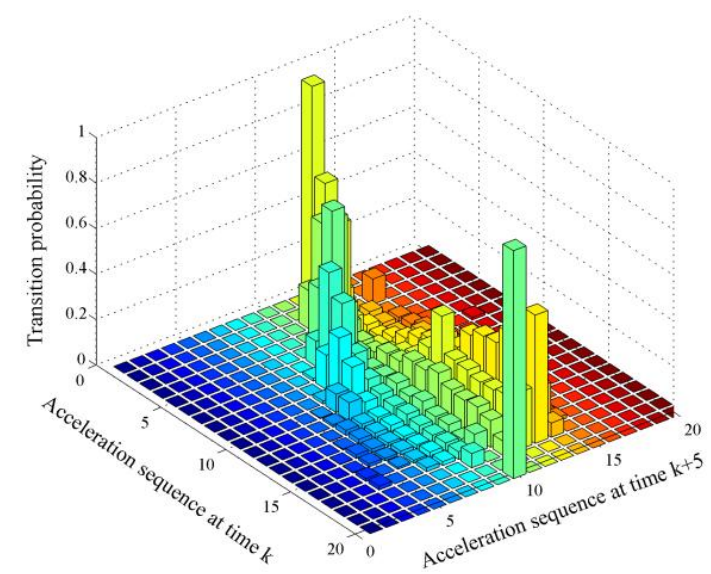

(a) Five-step acceleration transition probability.

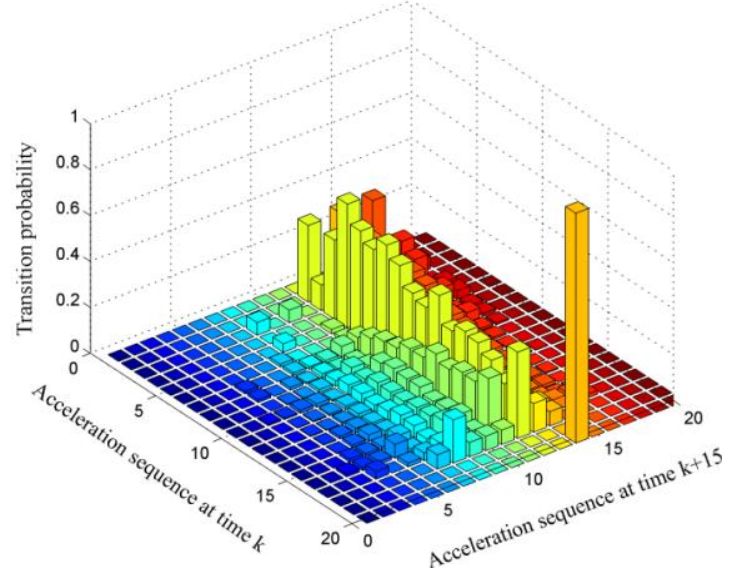

(c) Fifteen-step acceleration transition probability.

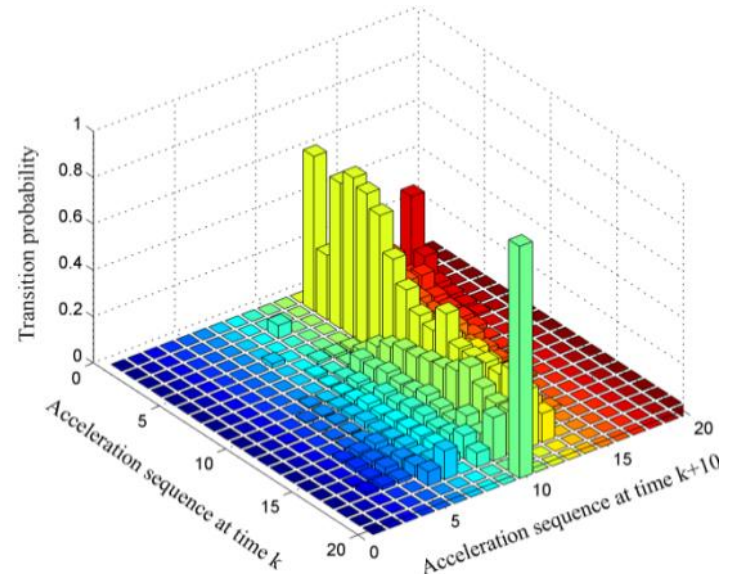

(b) Ten-step acceleration transition probability.

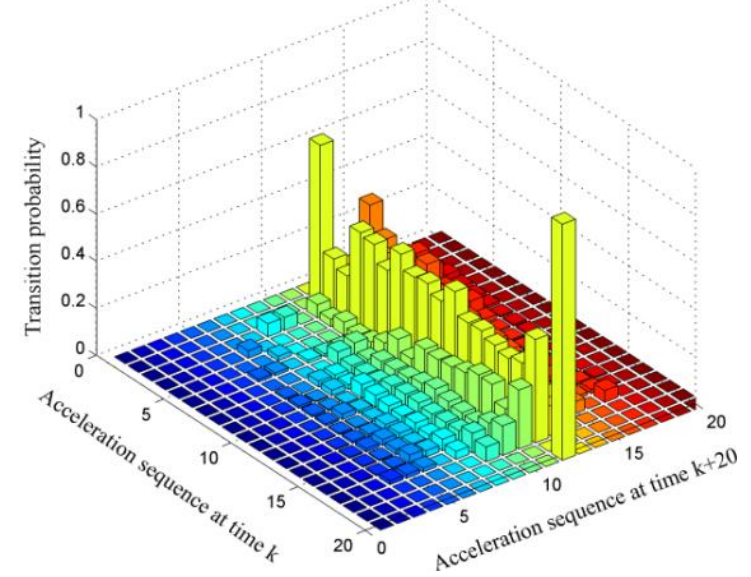

(d) Twenty-step acceleration transition probability.

Fig. 9. Examples of acceleration transition probabilities at a speed of $18 \mathrm{~km} \cdot \mathrm{h}^{-1}$.

The predicted speed can be calculated from the corresponding acceleration, which is looked up in the probability transition matrix. As an example, the acceleration transition probabilities at a speed of $18 \mathrm{~km} . \mathrm{h}^{-1}$ are depicted in Fig. 9. Note that the acceleration range is evenly divided into 20 discrete points. For this particular speed, it is apparent that the acceleration transition probability of a real driving always presents an irregular distribution, with increased preview horizon. Four of the time horizons, including $5 \mathrm{~s}, 10 \mathrm{~s}, 15 \mathrm{~s}$, and $20 \mathrm{~s}$, were chosen to investigate forecasting performance. Moreover, a jump of the acceleration transition probability can be observed in each subplot, indicating an obvious likelihood for the variation between two specific acceleration values in this urban driving cycle, given a vehicular velocity. To clearly illustrate the performance of the method, local predicted speeds (2000s-3500s) are plotted against real values, as shown in Fig. 10 (a), as well as the corresponding average forecasting errors over each horizon in four cases, shown in Fig. 10 (b). The prediction results become less accurate as the horizon increases, since under the Markov chain model the causal link between the current speed and predicted speed over a long term is almost not as strong as for a short-term case. In addition, quantitative results in Table 2 reveal the root-mean-squared-error (RMSE) values of the forecasting speeds for the preview horizon of $5 \mathrm{~s}, 10 \mathrm{~s} 15 \mathrm{~s}$ and $20 \mathrm{~s}$ reach $2.128 \mathrm{~km} . \mathrm{h}^{-1}, 5.331 \mathrm{~km} . \mathrm{h}^{-1}, 7.855 \mathrm{~km} . \mathrm{h}^{-1}$ and $9.670 \mathrm{~km} \cdot \mathrm{h}^{-1}$, demonstrating an increased prediction error as the preview horizon grows. 


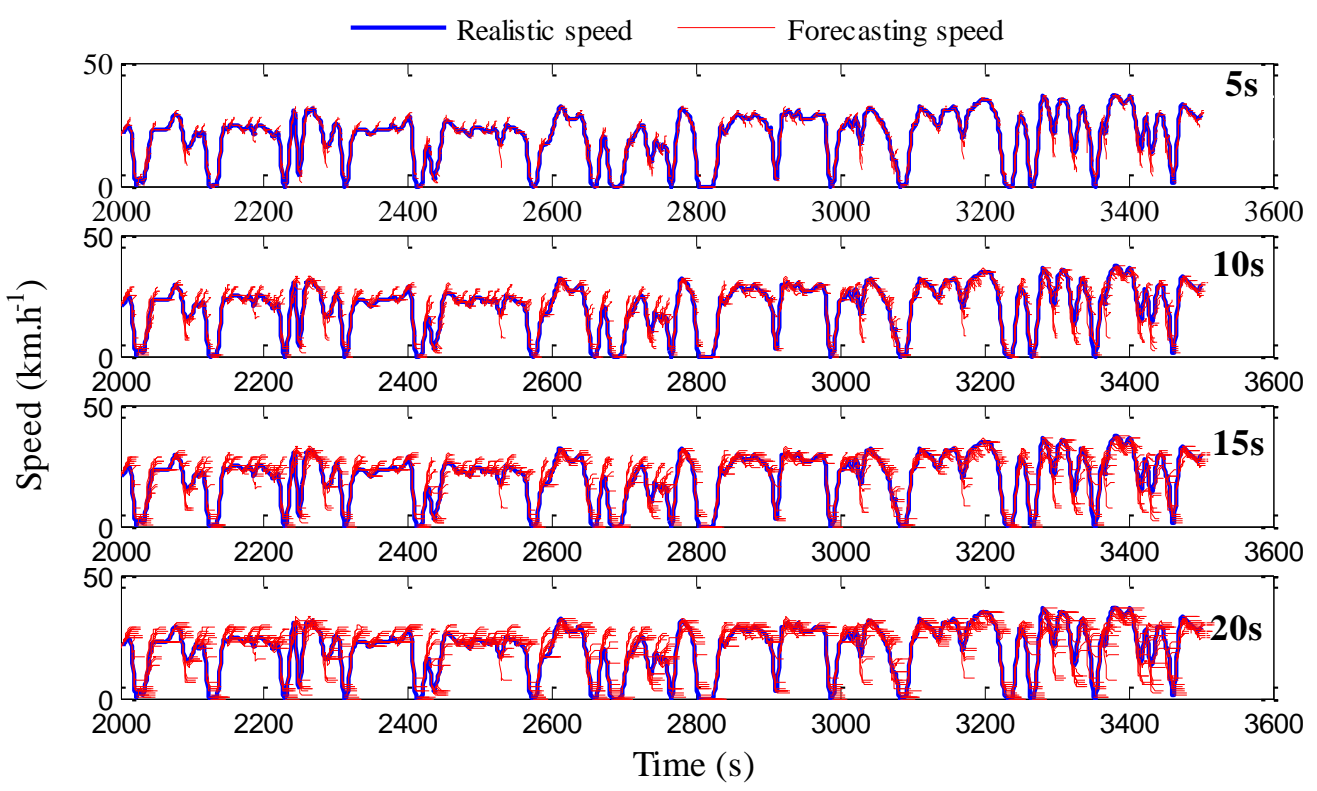

(a) Comparison between real and predicted speed.

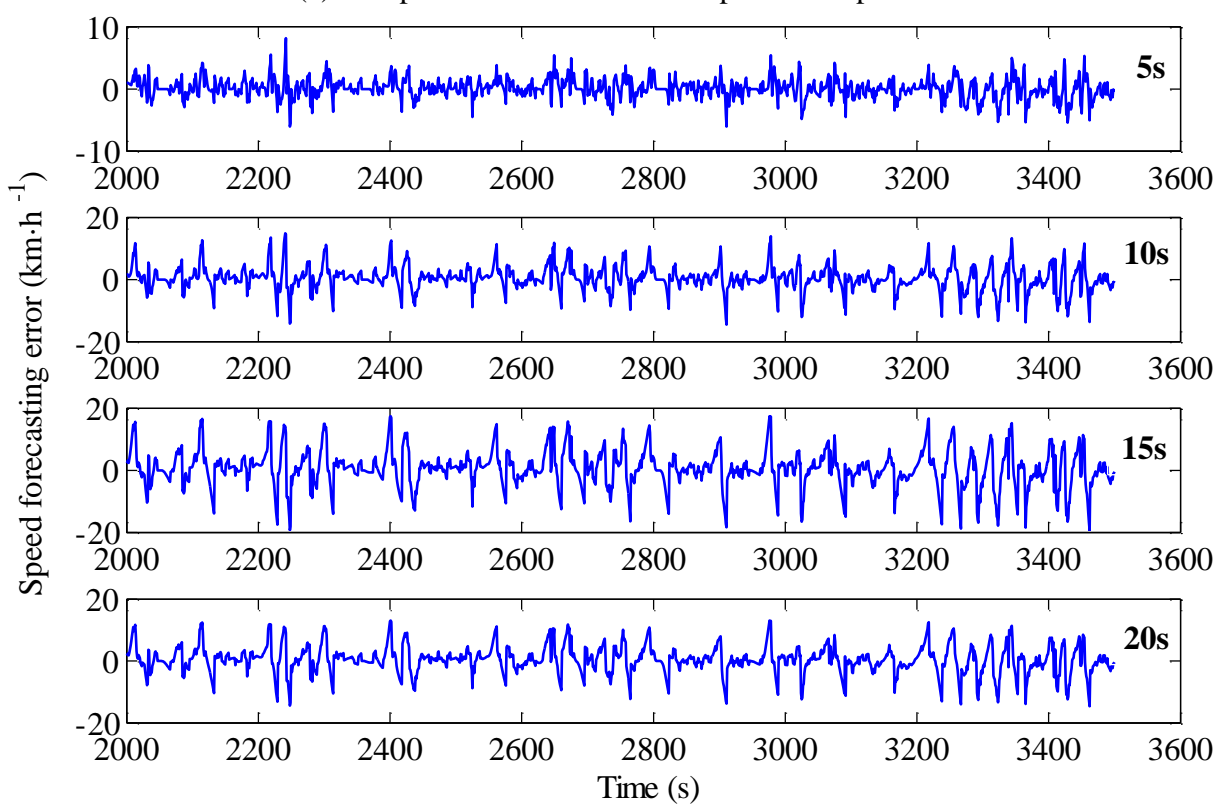

(b) Average speed forecasting over each preview horizon.

Fig. 10. Speed forecasting results (2000s - 3500s).

Table 2 RMSE values of the forecasting speed with four preview horizons

\begin{tabular}{c|c}
\hline Preview horizon length $(\mathbf{s})$ & RMSE $\left(\mathbf{k m} \cdot \mathbf{h}^{\mathbf{- 1}}\right)$ \\
\hline 5 & 2.128 \\
\hline 10 & 5.331 \\
\hline 15 & 7.855 \\
\hline 20 & 9.670 \\
\hline
\end{tabular}

\section{Reference SOC planning}

Unlike adaptive ECMS [25-26] in which the reference SOC is planned throughout an entire driving cycle, the reference SOC of MPC for PHEVs must be arranged over receding preview horizons. More specifically, upper and lower boundary SOCs must be defined for each preview horizon so that the DP or PMP method can be subsequently carried out. Assuming no power loss, if the bus is stationary at a bus stop or traffic light, and auxiliary power sources (e.g. 
electric air-conditioner) are neglected, the reference SOC can be dynamically tuned for each prediction horizon utilizing the predicted speed [23, 46]:

$$
S O C_{k+l_{\max }}^{r e f}=S O C_{k}-\frac{D_{k+l_{\max }}}{D_{\text {total }}-D_{k}}\left(S O C_{k}-S O C_{f}\right)
$$

where $k$ is the current time step, $l_{\max }$ is the length of the prediction horizon, $S O C_{k+l_{\max }}^{r e f}$ is the reference SOC at the end of the horizon, $S O C_{k}$ is the current SOC, $S O C_{f}$ is the preset final SOC, $D_{\text {total }}$ is the total driving distance, and $D_{k}$ denotes the distance travelled by the bus, which can be expressed as

$$
D_{k}=\int_{0}^{k \Delta t} v(t) d t
$$

where $v$ is the real speed and $\Delta t$ is the time step of $1 \mathrm{~s}$.

And the predicted driving distance $D_{k+l_{\max }}$ can be expressed as

$$
D_{k+l_{\max }}=\int_{k \Delta t}^{\left(k+l_{\max }\right) \Delta t} v_{p}(t) d t
$$

where $v_{p}$ is the predicted speed of each preview horizon.

\section{PMP-MPC and numerical solution}

\subsection{PMP-MPC framework}

The PMP-MPC strategy involves minimizing total energy consumption cost over each preview horizon. Therefore, the objective function can be defined as

$J=\min \int_{t_{k}}^{t_{k+l_{\max }}} L(\cdot) d t=\min \int_{t_{k}}^{t_{k+l_{\max }}}\left(p_{f} \dot{m}_{f}+p_{e} P_{b a t}\right) d t$

where $J$ is the total energy consumption cost, $L$ is the instantaneous energy consumption cost; $k$ is the $k$-th time step; $l_{\text {max }}$ is the length of the prediction horizon; $p_{f}$ and $p_{e}$ denote the fuel and electricity price, respectively; $\dot{m}_{f}$ is the EGU fuel rate; and $P_{b a t}$ is the total power consumption of the battery. Here, both the fuel and electricity consumption are included in the objective function, rather than only fuel usage [2, 25, 27, 48].

For the power-split controller, SOC is chosen as the state variable, and the state equation can be written as

$f(S O C)=S \dot{C} C=-\frac{U_{o c}-\sqrt{U_{o c}^{2}-4 R_{b} P_{b}}}{2 Q_{b} R_{b}}$

where $U_{o c}$ is the open circuit voltage and $Q_{b}$ is the battery capacity.

In addition, the EGU output power $P_{e g u}$ is selected as the control variable. Then, the Hamiltonian function can be given as

$H\left(S O C, P_{\text {egu }}, \lambda\right)=L\left(\right.$ SOC,$\left.P_{\text {egu }}\right)+\lambda f(S O C)$

where $\lambda$ is the co-state variable, which evolves according to the co-state dynamics governed by

$\dot{\lambda}=-\frac{\partial H}{\partial S O C}=-\lambda \frac{\partial S \dot{O} C}{S O C}$.

Moreover, the normal equation can be presented as

$S \dot{C} C=\frac{\partial H}{\partial \lambda}=f(S O C)$ 
where the state equation has the flowing expression:

$f(S O C)=-\frac{1}{Q_{b}} \cdot \frac{U_{o c}-\sqrt{U_{o c}^{2}-4 R_{b} P_{b}}}{2 R_{b}}$.

For the PMP method, the necessary condition takes the following formulation:

$H\left(\operatorname{SOC}^{*}, \lambda, P_{e g w}^{*}, t\right) \leq H\left(S O C, \lambda, P_{e g w}^{*}, t\right)$.

Furthermore, the constrained SOC boundaries should be respected when minimizing the Hamiltonian function at the $k$-th preview horizon. For the upper boundary value,

$S O C_{\text {intial }}=S O C_{k}^{0}$

and for the lower boundary value,

$S O C_{\text {end }}=S O C_{k+l_{\max }}^{f}$

where $S O C_{k}^{0}$ and $S O C_{k+l_{\max }}^{f}$ are the initial and final SOC values over the $k$-th prediction horizon determined by the reference SOC.

Then, the optimal control input can be captured by the expression:

$P_{e g u}^{*}=\operatorname{argmin} H\left(S O C, P_{e g u}, \lambda, t\right)$

For state variables, the evolving dynamics can be described as

$S O C_{k+1}=S O C_{k}+S O \dot{C} C_{k} d t$.

Further, the state variable has the following constraints:

$S O C_{\min } \leq S O C_{k} \leq S O C_{\max }$

where $S O C_{\min }$ and $S O C_{\max }$ denote the boundary SOCs of the battery.

Similarly, physical limitations imposed on power components can be given as

$\left\{\begin{array}{l}n_{m \_ \text {min }} \leq n_{m} \leq n_{m \_ \text {max }} \\ T_{e_{\_} \min } \leq T_{e} \leq T_{e \_ \text {max }} \\ T_{m \_\min } \leq T_{m} \leq T_{m \_ \text {max }} \\ P_{\text {egu_min }} \leq P_{\text {egu }} \leq P_{\text {ege_max }} \\ I_{b \_ \text {min }} \leq I_{b} \leq I_{b \_ \text {max }}\end{array}\right.$

where $T_{e}$ denotes the engine torque, $\min$ and $\max$ denote the upper and lower boundary values, respectively.

\subsection{Numerical solution}

\subsubsection{Shooting method}

For PMP-MPC, minimizing the energy consumption cost over the moving horizon results in a two-point-boundary-value problem (TPBVP). Therefore, the typical method, i.e., shooting method, is an effective approach for obtaining the numerical solution. Since a critical step of the shooting process is tuning the initial co-state values in order to hit a preset target SOC value as soon as possible, the secant method is introduced to modify the initial co-state value at the $k$-th prediction horizon [23]:

$\begin{cases}\lambda_{i}=\lambda_{0} & i=1 \\ \lambda_{i}=\lambda_{i-1}+\delta \quad & i=2 \\ \lambda_{i}=\lambda_{i-1}-\left(\lambda_{i-1}-\lambda_{i-2}\right) & \frac{S O C_{l_{\max }^{i-1}} \operatorname{SOC}_{l_{\max }^{i-1}-S O C_{l_{\max }}^{i-2}}^{f} i=3,4, \cdots, i_{\max }}{l_{\max }}\end{cases}$

where $i$ is the shooting sequence, $\lambda_{i}$ is the initial co-state value of each shooting, $\lambda_{i}(i=1,2)$ are the first two preset initial co-state values determined by constants $\lambda_{0}$ and $\delta, l_{\max }$ is the prediction horizon length, $i_{\max }$ is the maximum shooting 
time, and $S O C_{l_{\max }}^{f}$ is the final SOC value of each horizon computed by Eq. (5).

Furthermore, since the SOC interval between the upper and lower boundaries is always minor in each rolling horizon, a strengthened convergence factor, despite possibly improve solution accuracy, may lead to overshooting or non-convergent results. To avoid this phenomenon, the threshold for maximum shooting time $N_{s}$ in each preview horizon must be set:

$i_{\max } \leq N_{s}$

where $i_{\max }$ is the actual shooting time of each preview horizon.

\subsubsection{Flowchart of algorithm}

To further describe the proposed PMP-MPC method, a detailed flowchart of the algorithm is presented in Fig. 11, where vector $\boldsymbol{P}_{\text {egu }}$ denotes the allowable EGU power sequence, and $\varepsilon$ is the preset convergence factor.

The primary steps for implementing PMP-MPC are as follows:

Step 1: At the $k$-th step, forecast the speed sequence based on the Markov chain model.

Step 2: Compute the reference SOC according to Eq. 5.

Step 3: Conduct the shooting in the moving horizon $\left[k \Delta t\left(k+l_{\max }\right) \Delta t\right]$ until the final SOC meets the convergence condition or the terminal shooting time is triggered. Then, compute SOC derivative based on Eq. 9.

Step 4: Tune the initial co-state value in each horizon by implementing the shooting method and applying the secant method. The SOC and co-state variables evolve with the dynamic equations.

Step 5: Search the optimal power split relationship during each step by minimizing the Hamiltonian function, while following the physical constraints.

Step 6: Based on Step 5, obtain the optimal EGU output power and SOC sequence and choose the first element of the sequence.

Step 7: Refresh all current state variables according to the vehicle dynamics.

Step 8: Repeat steps 1-7. 


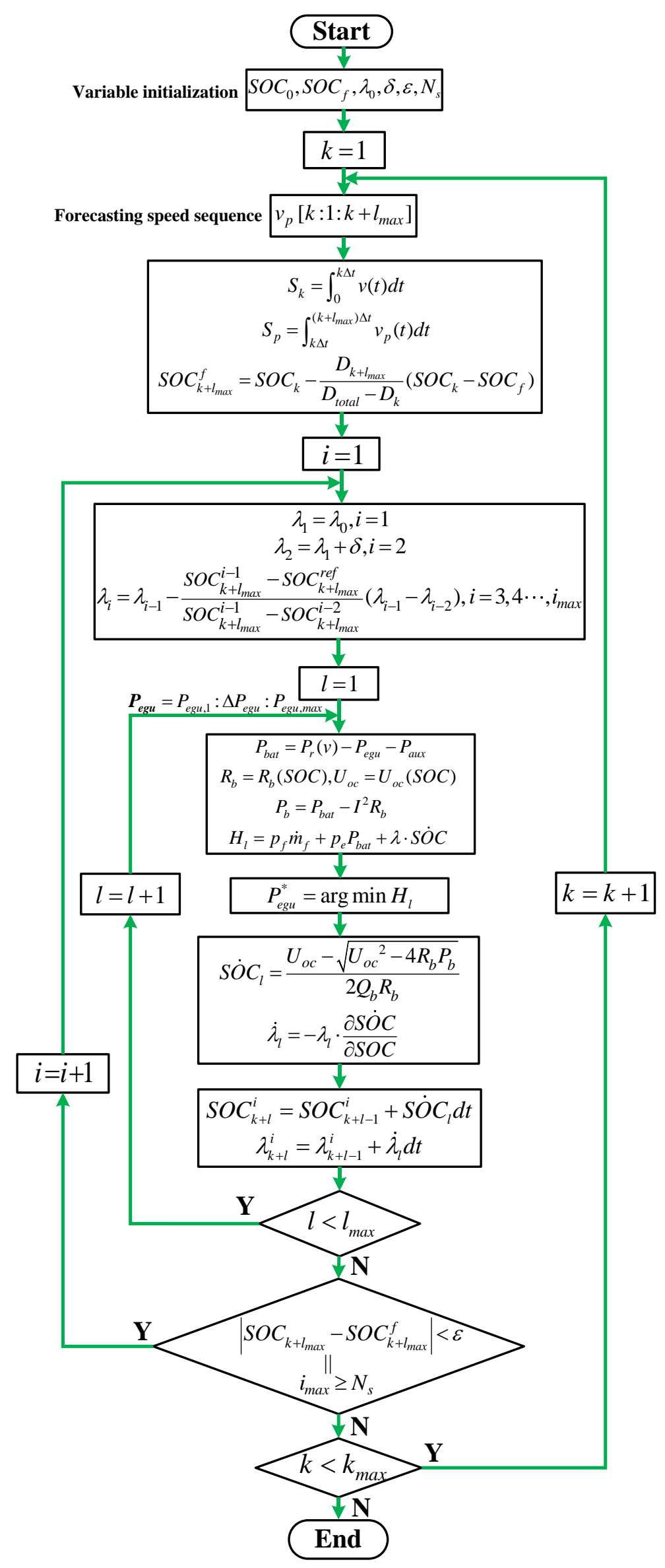

Fig. 11. Flowchart of PMP-MPC algorithm. 


\section{Results analysis and comparison}

The seventh speed profile, depicted in Fig. 8, has a net driving time of 4.52 hours and was used as an example to investigate PMP-MPC and DP-MPC based on four prediction horizons $(5 \mathrm{~s}, 10 \mathrm{~s}, 15 \mathrm{~s}$, and $20 \mathrm{~s})$. To simulate a typical fully-charged battery, the initial SOC was set to 0.9 , and the lower SOC boundary was specified as 0.3 . The initial co-state values, $\lambda_{0}$ and $\delta$, were set to -0.656 and 0.04 , respectively, and the terminal shooting time $N_{s}$ was set to 6 . The total distance for this bus route is $70 \mathrm{~km}$ on average, therefore, the value of $D_{\text {total }}$ was set as $71 \mathrm{~km}$ to avoid a negative denominator. The convergence factor $\varepsilon$ was set to $10^{-5}$. In addition, the price of the natural gas and electricity were 0.688 USD. ${ }^{-3}$ and 0.112 USD.kWh ${ }^{-1}$, respectively, based on market prices. Note that all the simulations were performed on a laptop computer with a CPU of 2.3-GHz frequency and an 8-GB memory.

\subsection{Results of PMP-MPC}

The SOC profiles yielded from the PMP-MPC method based on four preview horizons $(5 \mathrm{~s}, 10 \mathrm{~s}, 15 \mathrm{~s}$, and $20 \mathrm{~s})$ are presented in Fig. 12. Quantitative results are summarized in Table 3. The final SOC levels almost reached the boundary value (0.3) in each case, suggesting that the four cases consume a similar amount of electricity. As the prediction horizon increases, the total cost is slightly reduced (see Table 3). It can also be observed that as the preview horizon increases, computational time per second of driving cycle significantly increases. This may be a result of the time required for PMP-MPC calculations over longer horizons, which involves the speed prediction, iterative calculation of the SOC and co-state variables, as well as interpolations for obtaining battery internal resistance and open circuit voltage from look-up tables, which are more time-consuming over longer horizons. Hence, the case (horizon $=5 \mathrm{~s}$ ) seems to be a preferable solution with a very good balance between optimality and computational efficiency.

Table 3 Results of PMP-MPC based on four prediction horizons

\begin{tabular}{c|c|c|c|c|c}
\hline Horizon $(\mathbf{s})$ & $\left.\mathbf{F C} \mathbf{~ ( ~}^{\mathbf{3}}\right)$ & $\mathbf{E C}(\mathbf{k W h})$ & Final SOC & Total cost (USD) & Computational time per second (s) \\
\hline 5 & 5.09 & 38.37 & 0.3053 & 7.8000 & 0.0130 \\
\hline 10 & 5.01 & 38.67 & 0.3005 & 7.7776 & 0.0262 \\
\hline 15 & 4.97 & 38.89 & 0.2971 & 7.7744 & 0.0413 \\
\hline 20 & 4.96 & 38.88 & 0.2973 & 7.7664 & 0.0502 \\
\hline
\end{tabular}

Note: FC and EC are abbreviations for fuel consumption and electricity consumption, respectively.

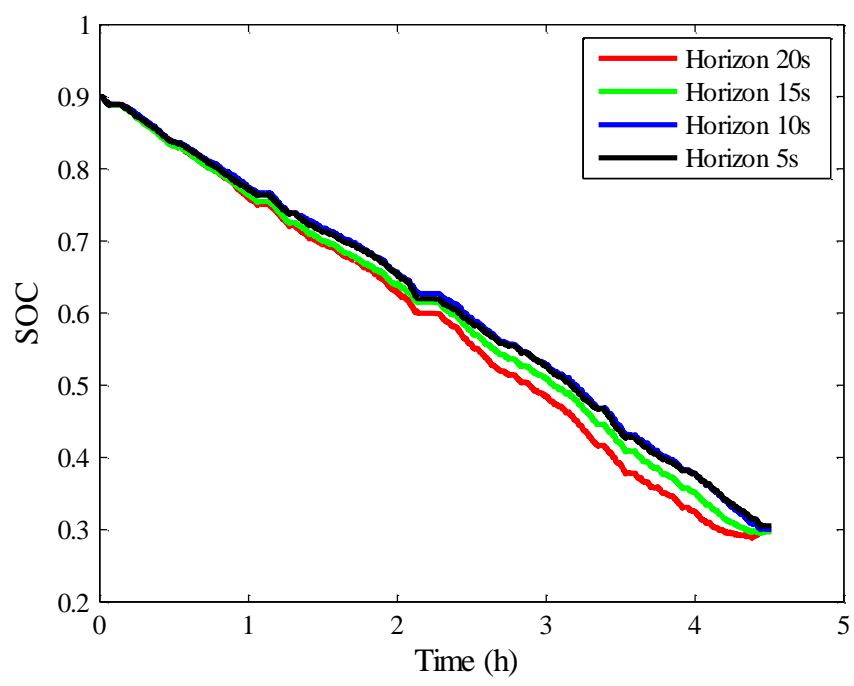

Fig. 12. SOC profiles of four prediction horizons using PMP-MPC. 

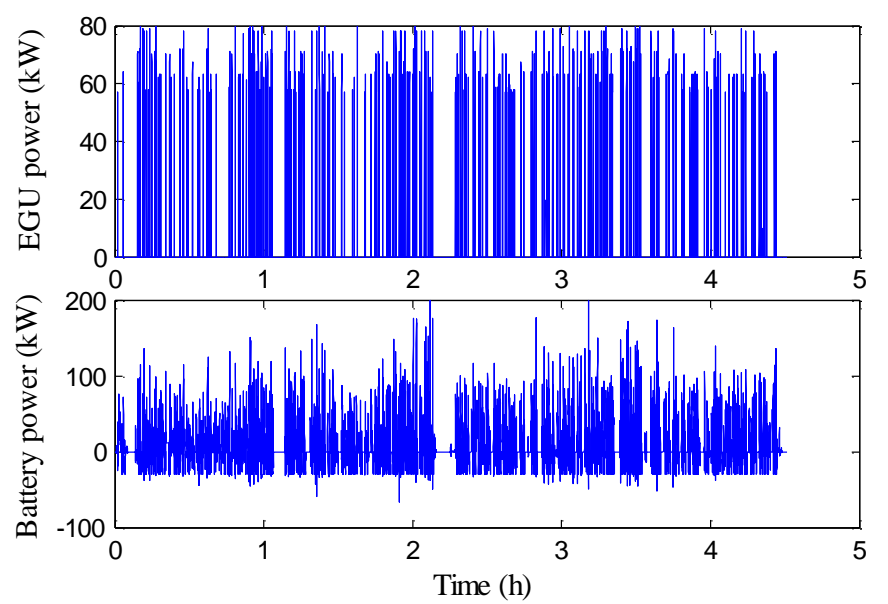

Fig. 13. Output power of EGU and battery (horizon $=5 \mathrm{~s}$ ).

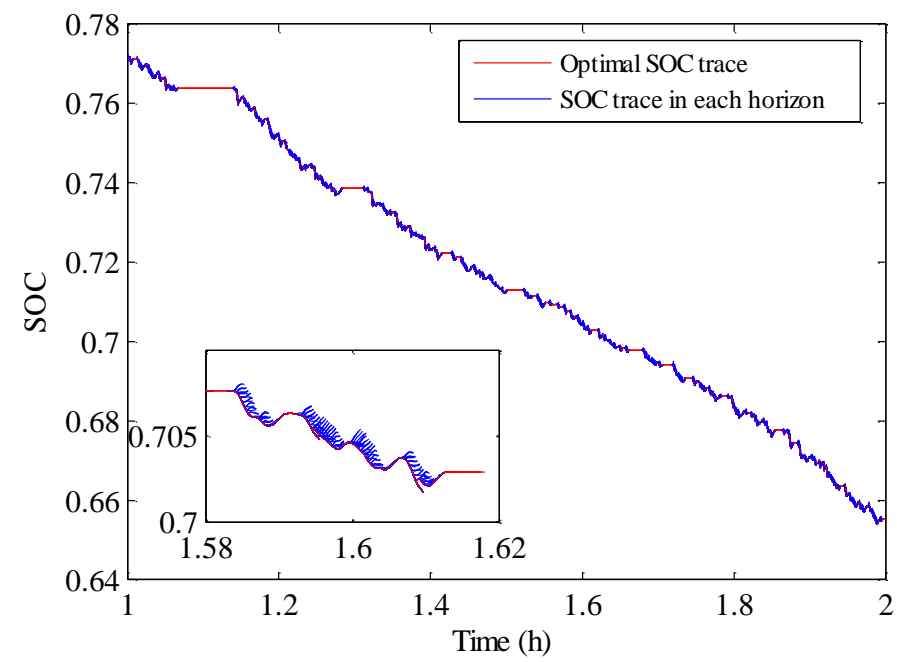

Fig. 14. Local SOC profiles (from $1 \mathrm{~h}$ to $2 \mathrm{~h}$ ) obtained using shooting method for each prediction horizon (horizon $=5 \mathrm{~s}$ ).

To further assess the PMP-MPC method, the power distribution between the EGU and battery over the 5s horizon are illustrated in Fig. 13. The blended discharging occurs throughout the entire trip rather than a CD-CS solution. In addition, we can observe the negative battery power, due to energy recuperation during braking when the electrical motor works as a generator. The local SOC profiles (from $1 \mathrm{~h}$ to $2 \mathrm{~h}$ ) generated via the shooting method are shown in Fig. 14. The red curve represents the result from the final shooting practice over each preview horizon, which acts as the optimal SOC trace, whereas the blue SOC trace represents an intermediate non-optimal solution during the shooting process.

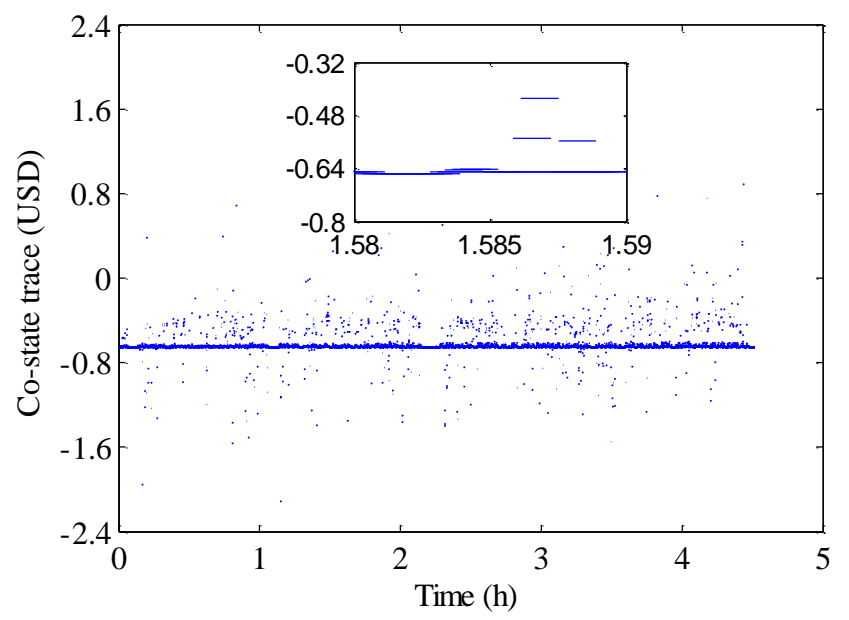

Fig. 15. Co-state traces obtained using the shooting method (horizon $=5 \mathrm{~s}$ ). 


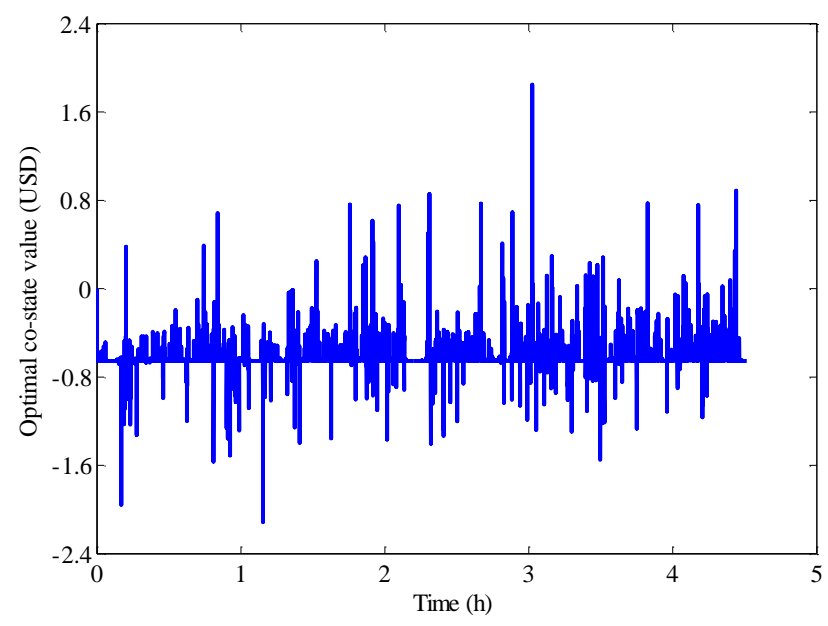

Fig. 16. Optimal initial co-state value (horizon $=5 \mathrm{~s}$ ).

The optimal co-state trace for the case of 5s prediction horizon is shown in Fig. 15, with a zoom-in view of the trace from $1.58 \mathrm{~h}$ to $1.59 \mathrm{~h}$. Fig. 16 depicts the associated optimal initial co-state. It can be observed that although the same initial co-state for all preview horizons was assigned, the optimal co-state determined by the shooting alters throughout the entire trip.

\subsection{Comparison with DP-MPC}

For comparison purposes, this section introduces the DP-MPC method, for which the iterative equation of the $k$-th prediction horizon can be expressed by [53]

$J_{l}^{*}\left(S O C_{h}\right)=\left\{\begin{array}{lr}\min _{q \in Q}\left[L\left(S O C_{h}, P_{e g u, q}\right)+J_{l+1}^{*}\right] & \left(l=l_{\max }-1, l_{\max }-2, \cdots, 1\right) \\ \min _{q \in Q}\left[L\left(S O C_{h}, P_{e g u, q}\right)+\varphi\left(S O C_{h}\right)\right] \quad\left(l=l_{\max }\right) & \end{array}\right.$

where $l$ denotes the $l$-th step in the prediction horizon; indices $h$ and $q$ are discrete points of battery SOC and EGU power, respectively; $L$ is a function of the instantaneous energy consumption cost; $J$ is the accumulated cost; $Q$ is the allowable set of index $q$; and $\varphi$ is a penalty function.

For a fair comparison, the same SOC boundaries were specified for DP-MPC and PMP-MPC. Computational time and numerical precision are strongly related to discretization scale of the battery SOC grid. Therefore, to obtain a precision comparable to that of PMP-MPC, the number of discrete SOC points in each preview horizon was set to 20 for DP-MPC. The SOC profiles are shown in Fig 17, and the results of energy consumption and computational time per second of the driving cycle are listed in Table 4. It can be seen that the calculation time increases with augmented prediction horizon, which can be attributed to the greater number of calculation steps over longer horizons. 


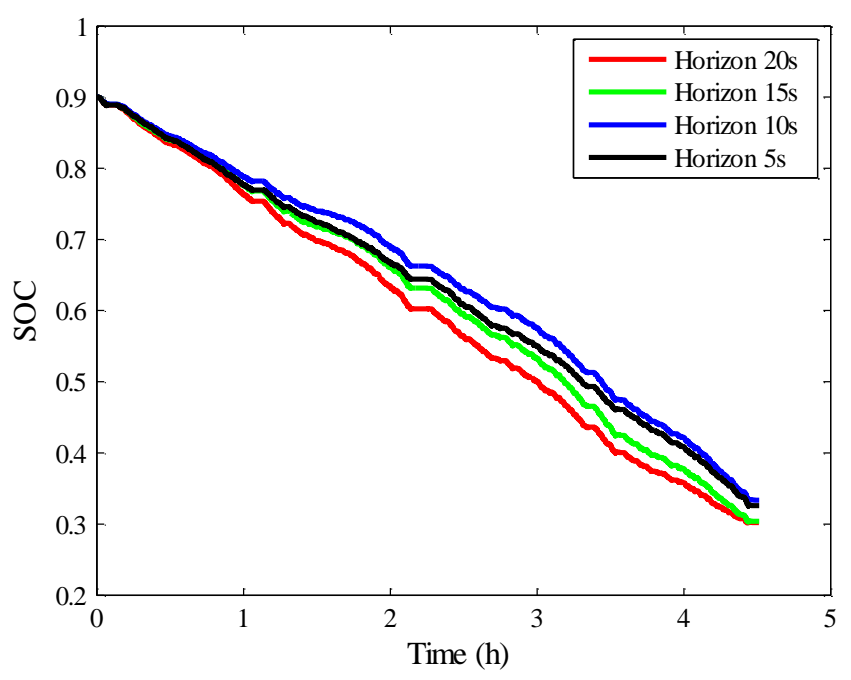

Fig. 17. SOC profiles of DP-MPC under four prediction horizons.

Comparing PMP-MPC with DP-MPC, it can be found that the former always exhibits a slight advantage in terms of numerical accuracy, mainly due to the accumulative error associated with estimating the cost-to-go value via the interpolation method for the DP algorithm. Computational efficiency of the PMP-MPC method is significantly higher than DP-MPC. The computation times of DP-MPC in all cases were at least four-fold greater. These large differences can be attributed to different optimization mechanisms. More specifically, massive interpolative and iterative calculations required by the DP to estimate cost-to-go values increase the computational time, whereas the PMP algorithm finds an optimal solution by instantaneously minimizing the Hamiltonian function using the shooting method and avoids interpolations, leading to higher numerical accuracy and computational efficiency. As a result, the DP-MPC inevitably needs a higher requirement for hardware resources, e.g., the main frequency or memory than the PMP-MPC for the online controller development, which can somewhat accelerate the controller aging or even weaken the production life.

To assess the effect of SOC discretization scale on the performance, the number of discrete SOC points was increased to 40 for each prediction horizon. The results are summarized in Table 5 and show that solution accuracy can be slightly improved. However, computational time increases dramatically, indicating that the interpolation of additional SOC grid points increases computational burden.

Table 4 Results of DP-MPC with four prediction horizons (SOC-20)

\begin{tabular}{c|c|c|c|c|c}
\hline Horizon $(\mathbf{s})$ & $\mathbf{F C}\left(\mathbf{m}^{\mathbf{3}}\right)$ & $\mathbf{E C}(\mathbf{k W h})$ & Final SOC & Total cost (USD) & Computational time per second (s) \\
\hline 5 & 5.44 & 37.04 & 0.3259 & 7.8912 & 0.0509 \\
\hline 10 & 5.62 & 36.53 & 0.3338 & 7.9584 & 0.1061 \\
\hline 15 & 5.09 & 38.47 & 0.3037 & 7.8112 & 0.1562 \\
\hline 20 & 5.07 & 38.57 & 0.3022 & 7.8080 & 0.2077 \\
\hline
\end{tabular}

Table 5 Results of DP-MPC with four prediction horizons (SOC-40)

\begin{tabular}{c|c|c|c|c|c}
\hline Horizon $(\mathbf{s})$ & $\mathbf{F C}\left(\mathbf{m}^{\mathbf{3}}\right)$ & $\mathbf{E C}(\mathbf{k W h})$ & Final SOC & Total cost (USD) & Computational time per second (s) \\
\hline 5 & 5.45 & 36.93 & 0.3275 & 7.8864 & 0.1375 \\
\hline 10 & 5.52 & 36.82 & 0.3293 & 7.9216 & 0.2382 \\
\hline 15 & 5.10 & 38.29 & 0.3065 & 7.7968 & 0.3086 \\
\hline 20 & 5.05 & 38.48 & 0.3036 & 7.7840 & 0.4049 \\
\hline
\end{tabular}

To further demonstrate the effectiveness of the proposed PMP-MPC, a combination of four typical standard driving cycles is used as a multi-cycle scenario, including two modal driving cycles - Chinese Urban Bus Driving Cycle (CUBDC) and Japanese 10-15 Exhaust Emission \& Fuel Economy Driving Schedule (JN1015), and two transient driving cycles - West Virginia City Driving Schedule (WVCITY) and Manhattan City Bus Driving Cycle (MANHATTAN). These standard driving cycles representing typical driving environments in different cities or countries have been broadly embodied into the commercial software such as ADVISOR [54], to evaluate the fuel 
economy or exhaust emissions. Fig. 18 shows the composite speed profile of the four driving cycles, where each transient driving cycle is intentionally arranged to follow a modal driving cycle to diversify the speed variations.

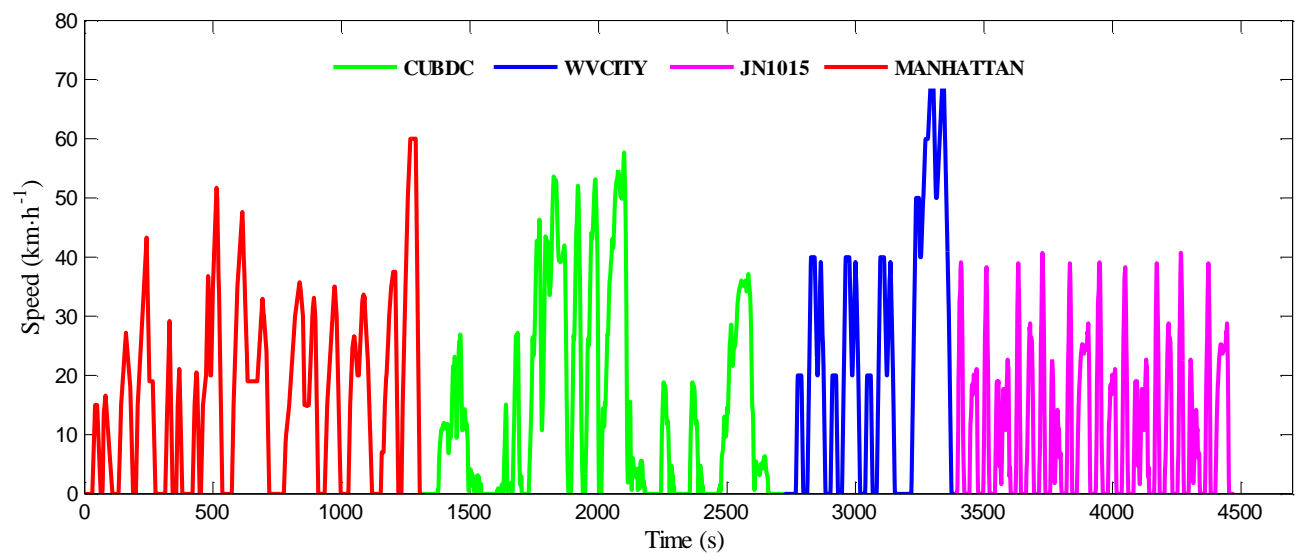

Fig. 18. A combination of four standard driving cycles.

Moreover, to imitate the driving mission for a plug-in electric city bus, a total of five consecutive composite driving cycles are used, with a total length of about $93.5 \mathrm{~km}$ and duration of $6.18 \mathrm{~h}$. The results of the DP-MPC and PMP-MPC are summarized in Table 6. It reveals that both the predictive methods reach the same total cost, whereas the PMP-MPC demonstrates a higher time-efficiency than the DP-MPC. In this case, we draw the same conclusion as the case of the city bus driving cycle. Moreover, the SOC profiles of the PMP-MPC and DP-MPC are shown in Fig 19 and Fig 20, respectively.

Table 6 Comparison of the PMP-MPC and DP-MPC for a multi-cycle scenario

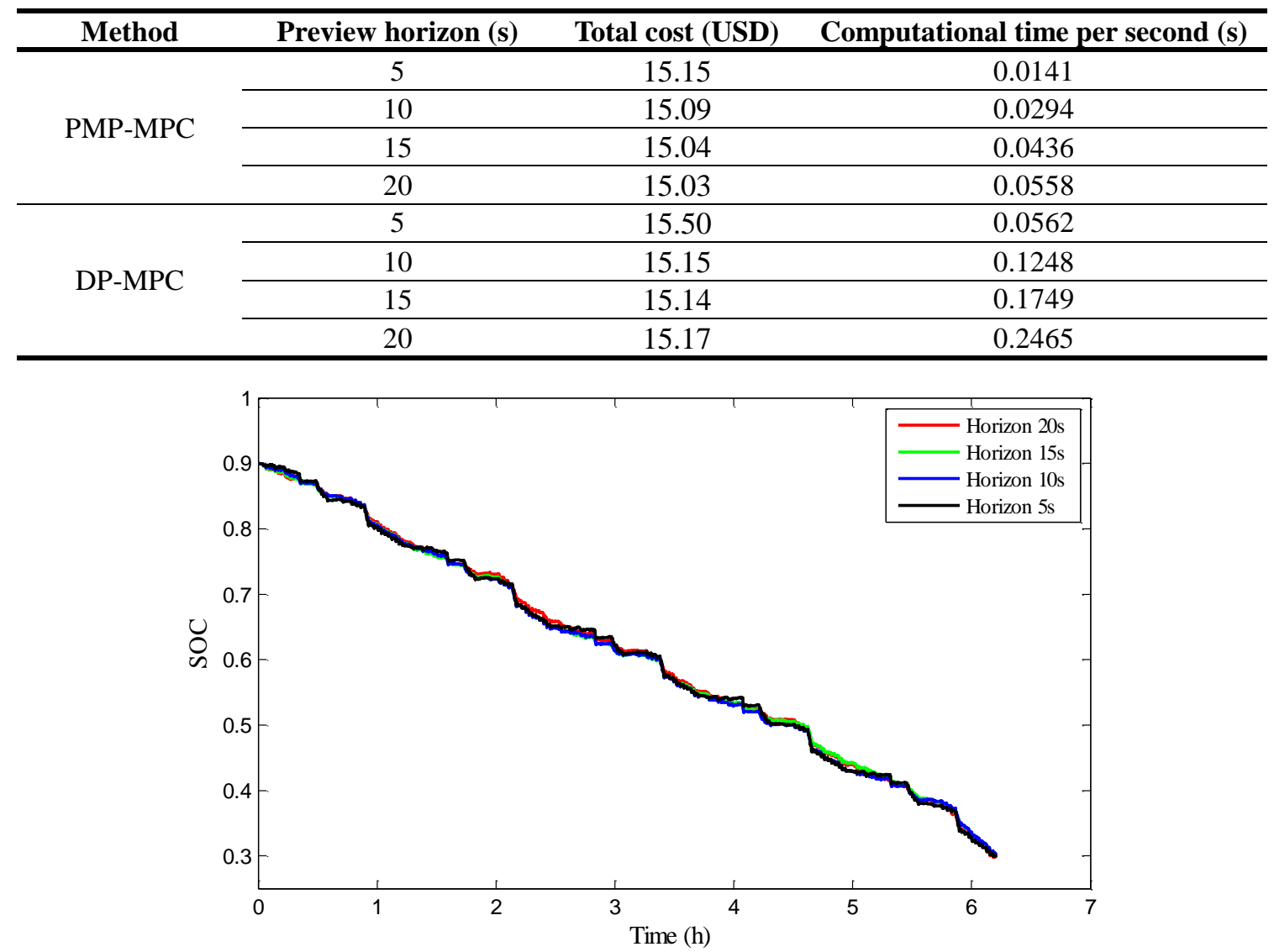

Fig. 19. SOC profiles of the PMP-MPC for a multi-cycle scenario. 


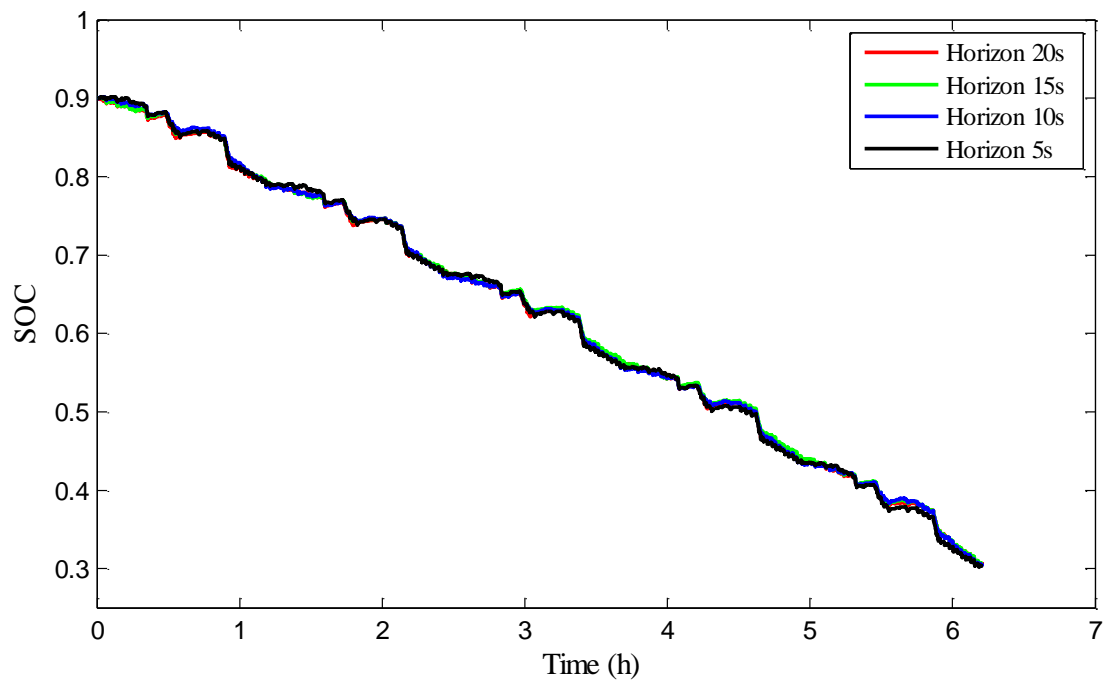

Fig. 20. SOC profiles of the DP-MPC for a multi-cycle scenario.

\subsection{Comparison of different methods}

Furthermore, both the DP-MPC and PMP-MPC methods are compared with DP and PMP methods, as well as the charging-depleting and charging-sustaining (CD-CS) method. For simulations, the number of discrete SOC points was set to 200 in order to obtain satisfactory precision using the DP algorithm.

The results demonstrate that the predictive methods (horizon $=5 \mathrm{~s}$ ) have higher fuel consumption and total cost than do global optimization methods, as shown in Table 7. The reason is that the MPC-based power management is essentially a local optimization algorithm. Moreover, the PMP method greatly reduces computational time, compared to the DP method, demonstrating higher computational efficiency. Overall, both the PMP and PMP-MPC algorithms have advantages over other methods, in computational efficiency, which shows that they may offer a superior choice for developing high-efficiency low-cost EMSs. Moreover, although the rule-based method, i.e., CD-CS, is capable of online implementation with fast computation, its energy cost is obviously higher.

Table 7 Simulation results of different methods

\begin{tabular}{|c|c|c|c|c|c|}
\hline \multirow{2}{*}{ Results } & \multicolumn{5}{|c|}{ Methods } \\
\hline & PMP-MPC & DP-MPC & DP & PMP & CD-CS \\
\hline Final SOC & 0.3053 & 0.3259 & 0.3032 & 0.3035 & 0.3373 \\
\hline $\mathrm{EC}(\mathrm{kWh})$ & 38.37 & 37.04 & 38.50 & 38.48 & 36.31 \\
\hline $\mathrm{FC}\left(\mathrm{m}^{3}\right)$ & 5.09 & 5.44 & 4.42 & 4.37 & 8.56 \\
\hline Total cost (USD) & 7.8000 & 7.8912 & 7.3520 & 7.3152 & 9.9568 \\
\hline Computational time per second (s) & 0.0130 & 0.0509 & 0.0943 & 0.0017 & 0.0002 \\
\hline Real-time applicability (N/A) & A & $\mathrm{A}$ & $\mathrm{N}$ & $\mathrm{N}$ & $\mathrm{A}$ \\
\hline
\end{tabular}




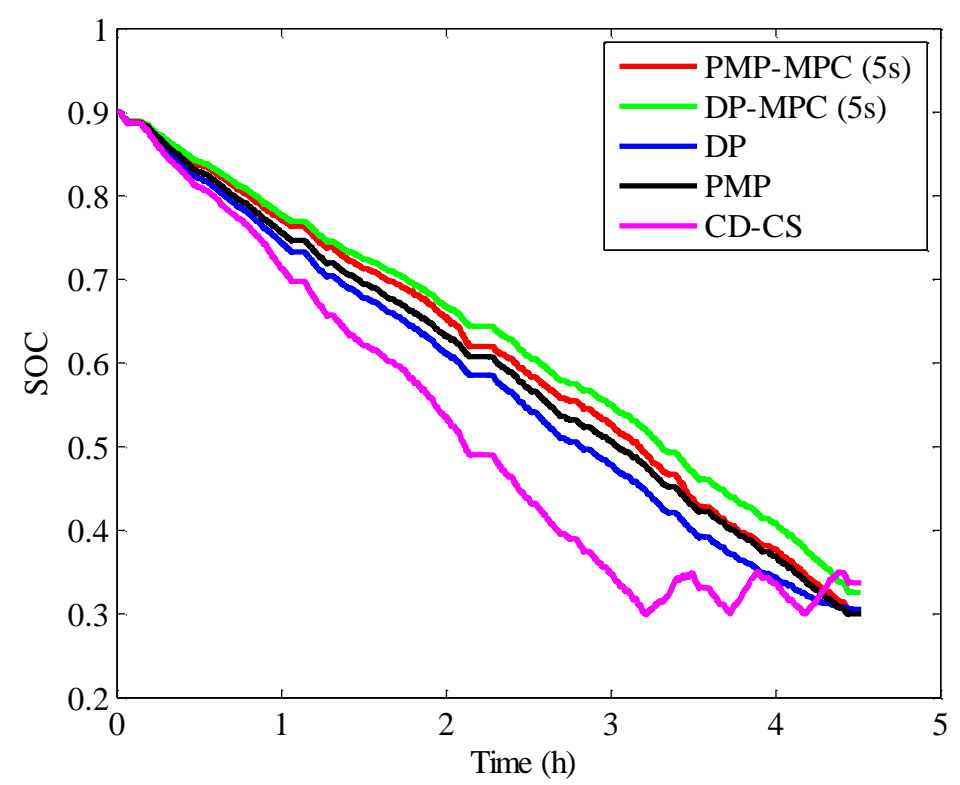

Fig. 21. SOC profiles of different methods (Preview horizon $=5 \mathrm{~s}$ for PMP-MPC and DP-MPC).

Fig. 21 shows the SOC profiles of the five methods. The rule-based method clearly undergoes a CD stage followed by a CS stage, with a trend that differs from the other methods. The SOC curves of all other methods show an analogous trend.

The PMP shooting process for the PMP controller is detailed in Fig. 22, where six shootings are triggered to target the lower SOC boundary. Fig. 23 illustrates the initial co-state value of each shooting. The optimal co-state value increases gradually before finally reaching a stable level. The corresponding optimal co-state trace is presented in Fig. 24.

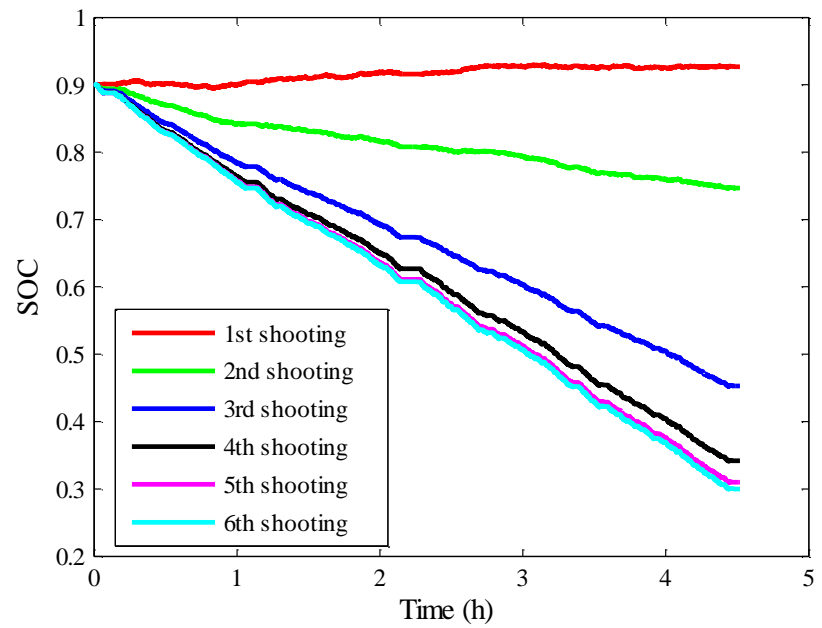

Fig. 22. SOC profiles generated by shooting method. 


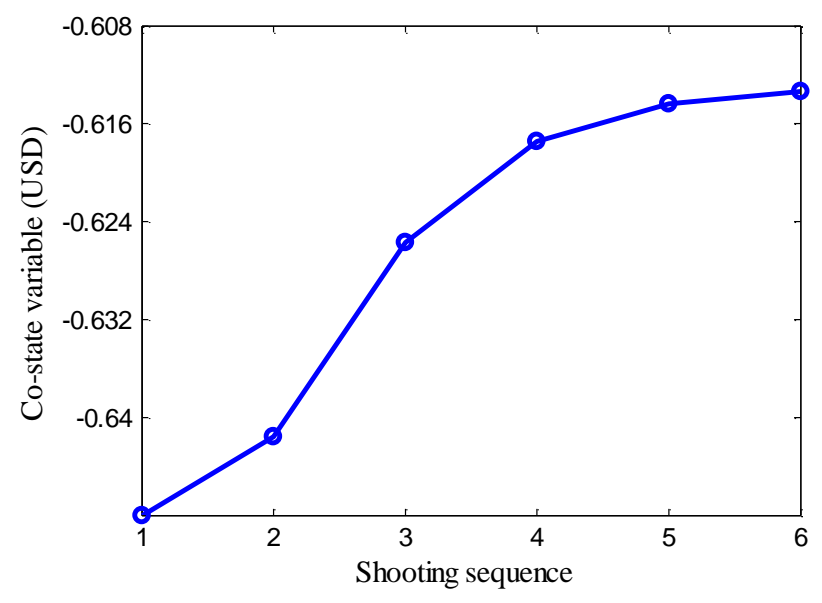

Fig. 23. Initial co-state value of each shooting process.

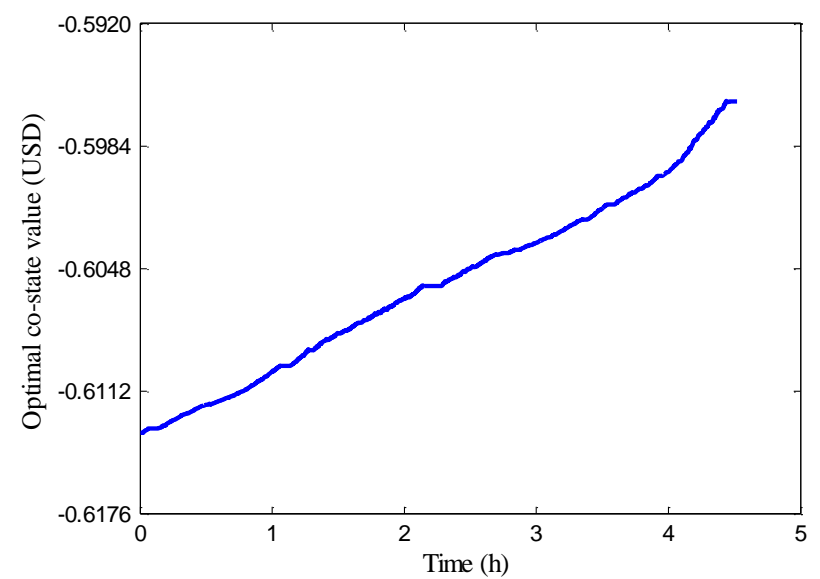

Fig. 24. Optimal co-state trace.

\section{Conclusions}

In this paper, a framework for implementing model predictive control based on Pontryagin's Minimum Principle is presented, in order to develop a time-efficient online energy management strategy for plug-in hybrid electric vehicles. The proposed PMP-MPC method exhibits a significant advantage over DP-MPC in terms of computational efficiency. Moreover, the equivalent factor does not need to be dynamically tuned in the PMP-MPC, which differs from widely used Equivalent Consumption Minimum Strategy-based predictive strategies. The comparison of the PMP-MPC and DP-MPC with four prediction horizons $(5 \mathrm{~s}, 10 \mathrm{~s}, 15 \mathrm{~s}$ and $20 \mathrm{~s})$ demonstrates that the calculation of the PMP-MPC is more than three times faster. A relatively short preview horizon is advised to meet a good balance between optimality and computational load. In the case of a preview horizon of $5 \mathrm{~s}$, the PMP-MPC has a total cost of 7.80 USD, and its computational time per step is 0.0130 s. Comparisons with common control practice, e.g., dynamic programming, Pontryagin's Minimum Principle, and charge-depleting-charge-sustaining strategy, demonstrate that the PMP-MPC produces a comparable total cost to the globally optimal solutions, $(6.1 \%$ and $6.6 \%$ deviations from DP and PMP, respectively), but with more than six times higher computational speed, compared to dynamic programming. Therefore, the proposed PMP-MPC proves to be a viable and attractive alternative for online predictive energy management of plug-in hybrid electric vehicles.

The future research work could focus on the model predictive control of energy management for plug-in hybrid electrical vehicles in the context of vehicle-to-vehicle and vehicle-to-infrastructure communication.

\section{Acknowledgements}

This work was supported in part by the EU-funded Marie Sklodowska-Curie Individual Fellowships (IF) Project under Grant 706253-pPHEV-H2020-MSCA-IF-2015, and in part by the Fundamental Research Funds for the Central Universities of China (Grant no. 310822151026, 310822151121, 106112016CDJXZ338825, 


\section{References}

1. Liu T, Hu X, Li S, Cao D. Reinforcement learning optimized look-ahead energy management of a parallel hybrid electric vehicle. IEEE/ASME Trans on Mechatronics 2017, 22(4): 1497-1507.

2. Onori S, Tribioli L. Adaptive Pontryagin's Minimum Principle supervisory controller design for the plug-in hybrid GM Chevrolet Volt. Appl Energy 2015;147:224-234.

3. Martinez C M, Hu X, Cao D, Velenis E, Gao B, Wellers M. Energy management in plug-in hybrid electric vehicles: Recent progress and a connected vehicles perspective. IEEE Trans Veh Technol 2017, 66(6): 4534-4549.

4. Xiong R, Duan Y, Cao J, Yu Q. Battery and ultracapacitor in-the-loop approach to validate a real-time power management method for an all-climate electric vehicle. Appl Energy 2018;217:153-165.

5. Gonder J, Markel T. Energy management strategies for plug-In hybrid electric vehicles. SAE Technical Paper, 2007, no.2007-01-0290.

6. Schouten N J, Salman M A, Kheir N A. Fuzzy logic control for parallel hybrid vehicles. IEEE Trans Contr Syst Technol 2002;10(3):460-468.

7. Lin C C, Peng H, Grizzle J W, Kang J M. Power management strategy for a parallel hybrid electric truck. IEEE Trans Contr Syst Technol 2003;11(6):839-849.

8. Li L, Yang C, Zhang Y, Zhang L, Song J. Correctional DP-based energy management strategy of plug-in hybrid electric bus for city-bus route. IEEE Trans Veh Technol 2015;64(7):2792-2803.

9. Serrao L, Onori S, Rizzoni G. ECMS as a realization of Pontryagin's minimum principle for HEV control. In: American Control Conference (ACC), 2009. p.3964-3969.

10. Chen Z, Xiong R, Wang C, Cao J. An on-line predictive energy management strategy for plug-in hybrid electric vehicles to counter the uncertain prediction of the driving cycle. Appl Energy 2017;185:1663-1672.

11. Xie S, Hu X, Qi S, Lang K. An artificial neural network-enhanced energy management strategy for plug-in hybrid electric vehicles. Energy 2018;163:837-848.

12. Chen Z, Mi C C, Xia B, You C. Energy management of power-split plug-in hybrid electric vehicles based on simulated annealing and Pontryagin's minimum principle. J Power Sources 2014;272:160-168.

13. Xiong R, Cao J, Yu Q. Reinforcement learning-based real-time power management for hybrid energy storage system in the plug-in hybrid electric vehicle, Appl Energy 2018;211:538-548.

14. Pisu P, Rizzoni G. A comparative study of supervisory control strategies for hybrid electric vehicles. IEEE Trans Contr Syst Technol 2007; 15(3):506-518.

15. Serrao L, Onori S, Rizzoni G. A comparative analysis of energy management strategies for hybrid electric vehicles. J Dyn Syst-T ASME 2011;133:031012.

16. He H, Tang H, Wang X. Global optimal energy management strategy research for a plug-in series-parallel hybrid electric bus by using dynamic programming. Math Probl Eng 2013, Article ID 708261.

17. Johannesson L, Asbogard M, Egardt B. Assessing the potential of predictive control for hybrid vehicle powertrains using stochastic dynamic programming. IEEE Trans Intell. Transp Syst 2007;8(1):71-83.

18. Moura S J, Fathy H K, Callaway D S, Stein J L. A stochastic optimal control approach for power management in plug-in hybrid electric vehicles IEEE Trans Contr Syst Technol 2011;19(3):545-555.

19. Kim N W, Lee D H, Zheng C, Shin C, Seo H, Cha S W. Realization of PMP-based control for hybrid electric vehicles in a backward-looking simulation. Int J Automot Technol 2014;15(4): 625-635.

20. Zhang S, Xiong R, Zhang C. Pontryagin's minimum principle-based power management of a dual-motor-driven electric bus. Appl Energy 2015;159:370-380.

21. Kim N, Cha S, Peng H. Optimal control of hybrid electric vehicles based on Pontryagin's minimum principle. IEEE Trans. Contr Syst Technol 2011;19(5):1279-1287.

22. Hou C, Ouyang M, Xu L, Wang H. Approximate Pontryagin's minimum principle applied to the energy management of plug-in hybrid electric vehicles. Appl Energy 2014;115:174-189.

23. Xie S, Li H, Xin Z, Liu T, Wei L. A Pontryagin minimum principle based adaptive equivalent consumption minimum strategy for a plug-in hybrid electric bus on a fixed route. Energies 2017;10(9):1379-1399.

24. Musardo C, Rizzoni G, Guezennec Y, Staccia B. A-ECMS: an adaptive algorithm for hybrid electric vehicle energy management. Euro J Contr 2005;11(4):509-524.

25. Sivertsson M, Eriksson L. Design and evaluation of energy management using map-based ECMS for the PHEV benchmark. Oil Gas Sci. Technol 2015;70(1):195-211.

26. Sun C, Hu X, Moura S J, Sun F. Velocity predictors for predictive energy management in hybrid electric vehicles. IEEE Trans. Contr Syst. Technol 2015; 23(3):1197-1204.

27. Borhan H A, Vahidi A. Model predictive control of a power-split hybrid electric vehicle with combined battery and ultracapacitor energy storage In: American Control Conference (ACC), 2010, pp.5031-5036.

28. Gu B, Rizzoni G. An adaptive algorithm for hybrid electric vehicle energy management based on driving pattern recognition. In: Proc. ASME Mech Eng. Congr. and Exp, 2006,pp.249-258.

29. Yokoi Y, Ichikawa S, Doki S, Okuma S. Driving pattern prediction for an energy management system of hybrid electric vehicles in a specific driving course. In Proc 30th Conf IEEE Ind and Elect, 2004, pp.1727-1732.

30. Borhan H, Vahidi A, Phillips A M, Kuang M L, Kolmanovsky I V, Cairano S D. MPC-based energy management of a power-split hybrid electric 
vehicle. IEEE Trans. Contr Syst Technol 2012;20(3):593-603.

31. Guo L, Gao B, Gao Y, Chen H. Optimal energy management for HEVs in eco-driving applications using bi-Level MPC. IEEE Trans Intell Transp Syst 2017;18(8):2153-2162.

32. Vajedi M, Azad N L. Ecological adaptive cruise controller for plug-in hybrid electric vehicles using nonlinear model predictive control. IEEE Trans Intell Transp Syst 2015;17(1):113-122.

33. Shen D, Bensch V, Miiller S. Model predictive energy management for a range extender hybrid vehicle using map information IFAC-PapersoOnline 2015;48(15):263-270.

34. Zeng X, Wang J. A parallel hybrid electric vehicle energy management strategy using stochastic model predictive control with road grade preview. IEEE Trans Contr Syst Technol 2015; 23(6):2416-2423.

35. Li G, Zhang J, He H. Battery SOC constraint comparison for predictive energy management of plug-in hybrid electric bus. Appl Energy 2017; 194:578-587.

36. Sun C, Moura S J, Hu X, Hedrick J K, Sun F. Dynamic traffic feedback data enabled energy management in plug-in hybrid electric vehicles. IEEE Trans. Control Syst. Technol 2015;23(3):1075-1086.

37. Sun C, Sun F, He H. Investigating adaptive-ECMS with velocity forecast ability for hybrid electric vehicles. Appl Energy 2017, 185:1644-1653.

38. Unger J, Kozek M, Jakubek S. Nonlinear model predictive energy management controller with load and cycle prediction for non-road HEV. Contr Eng Pract 2015;36:120-132.

39. Ebbesen S, Salazar M, Elbert P, Bussi C, Onder C H. Time-optimal control strategies for a hybrid electric race car. IEEE Trans Contr Syst Technol 2018, 26(1):233-247.

40. Zhang F, Xi J, Langari R. Real-time energy management strategy based on velocity forecasts using V2V and V2I communications. IEEE Trans Intell Transp Syst 2017;18(2):416-430.

41. Wen C T, Ma X Y, Ydstie B E. Analytical expression of explicit MPC solution via lattice piecewise affline function. Automatica 2009;45(4):910-917.

42. Taghavipour A, Azad N L, McPhee J. Real-time predictive control strategy for a plug-in hybrid electric powertrain. Mechatronics 2015;29:13-27.

43. Zhang J, Shen T. Real-time fuel economy optimization with nonlinear MPC for PHEVs IEEE Trans. Contr Syst Technol 2016;24(6):2167-2175

44. Xie S, He H, Peng J. An energy management strategy based on stochastic model predictive control for plug-in hybrid electric buses. Appl. Energy 2017;196:279-288.

45. Li L, You S, Yang C, Yan B, Song J, Chen Z. Driving-behavior-aware stochastic model predictive control for plug-in hybrid electric buses. Appl Energy 2016;162:868-879.

46. Xie S, Hu X, Xin Z, Li L. Time-efficient stochastic model predictive energy management for a plug-in hybrid electric bus with adaptive reference state-of-charge advisory. IEEE Trans Veh Technol, 2018;67(7):5671-5682.

47. Cairano S D, Bernardini D, Bemporad A, Kolmanovsky I V. Stochastic MPC with learning for driver-predictive vehicle control and its application to HEV energy management. IEEE Trans Contr SystTechnol 2014;22(3):1018-1031.

48. Hemi H, Ghouili J, Cheriti A. Combination of Markov chain and optimal control solved by Pontryagin's Minimum Principle for a fuel cell/supercapacitor vehicle. Energy Convers Manage 2015;91:387-393.

49. Hu X, Martinez C M, Yang Y. Charging, power management, and battery degradation mitigation in plug-in hybrid electric vehicles: A unified cost-optimal approach. Mech Syst Signal Pr 2017;87:4-16.

50. Bashash S, Moura S J, Forman J C, Fathy H K. Plug-in hybrid electric vehicle charge pattern optimization for energy cost and battery longevity. J Power Sources 2011;196(1):541-549.

51. Wang J, Xie S, Liu X, Yuan Y, Li B, Li S. Research and development of a chassis for the extended-range and plug-in commercial vehicle. Shaanxi Automobile Group, Xi'an, China, Res. Rep., 2015.

52. Zou C, Zhang L, Hu X, Wang Z, Wik T, Pecht M. A review of fractional-order techniques applied to lithium-ion batteries, lead-acid batteries, and supercapacitors. J Power Sources 2018;390:286-296.

53. Liu J, Peng H. Modeling and control of a power-split hybrid vehicle. IEEE Trans. Control Syst. Technol., 2008;16(6):1242-1251.

54. T Markel, A Brooker, T Hendricks, V Johnson, K Kelly, B Kramer, M O'Keefe, S Sprik, K Wipke. ADVISOR: a systems analysis tool for advanced vehicle modeling, J Power Sources, 2002;110(2):255-266 
2018-12-17

Pontryagin's Minimum Principle based model predictive control of energy management for a plug-in hybrid electric bus

\author{
Xie, Shaobo
}

Elsevier

Xie S, Hu X, Xin Z, Brighton J. (2019) Pontryagin's Minimum Principle based model predictive control of energy management for a plug-in hybrid electric bus. Applied Energy, Volume 236,

February 2019, pp. 893-905

https://doi.org/10.1016/j.apenergy.2018.12.032

Downloaded from Cranfield Library Services E-Repository 